\title{
Selective Imaging of Extended Reflectors in Two-Dimensional Waveguides*
}

\author{
Chrysoula Tsogka ${ }^{\dagger}$, Dimitrios A. Mitsoudis ${ }^{\ddagger}$, and Symeon Papadimitropoulos ${ }^{\dagger}$
}

\begin{abstract}
We consider the problem of selective imaging extended reflectors in waveguides using the response matrix of the scattered field obtained with an active array. Selective imaging amounts to being able to focus at the edges of a reflector, which typically give rise to weaker echoes than those coming from its main body. To this end, we propose a selective imaging method that uses projections on low-rank subspaces of a weighted modal projection of the array response matrix, $\widehat{\mathbb{P}}(\omega)$. We analyze theoretically our imaging method for a simplified model problem where the scatterer is a vertical one-dimensional perfect reflector. In this case, we show that the rank of $\widehat{\mathbb{P}}(\omega)$ equals the size of the reflector divided by the cross-range array resolution. We also derive analytic expressions for the singular vectors of $\widehat{\mathbb{P}}(\omega)$ and carry out a detailed theoretical analysis of our selective imaging functional. Our numerical simulations are in very good agreement with the theory and illustrate the robustness of our imaging functional for reflectors of various shapes.
\end{abstract}

Key words. array imaging, waveguides, subspace projection method

AMS subject classifications. 35R30, 74J20, 74J25

DOI. $10.1137 / 130924238$

1. Introduction. In this work we consider the problem of detecting and imaging extended reflectors submerged in the sea, using acoustic waves produced by an active array. The array consists of $N$ transducers that act as sources and receivers. The data that we are going to use for imaging is assumed to be given in the form of the so-called array response matrix in the frequency domain, i.e., an $N \times N$ complex matrix whose entries are the Fourier transforms of the time traces of the echoes recorded at all receivers when each source emits a signal. The term "extended" refers to reflectors which are comparable in size to the acoustic wavelength. Here, the sea is modeled as an acoustic waveguide consisting of a single homogeneous water layer confined above by the sea surface and below by the seafloor, both assumed to be horizontal. Thus, our waveguide is an infinite strip of constant depth.

The imaging problem that we wish to solve is the following: Assuming that the extended reflector is illuminated by an active vertical array which spans the whole depth of the waveguide, and that the array response matrix is known, we want to image an extended reflective scatterer located in the waveguide. To this end we define a search domain (a bounded subset

\footnotetext{
* Received by the editors June 10, 2013; accepted for publication (in revised form) September 6, 2013; published electronically December 19, 2013. The first two authors' work was partially supported by the European Research Council under the European Union's Seventh Framework Programme (FP7/2007-2013)/ERC grant agreement 239959. The second author's work was also supported by the European Union's Seventh Framework Programme (FP7-REGPOT-2009-1) under grant 245749 through ACMAC.

http://www.siam.org/journals/siims/6-4/92423.html

${ }^{\dagger}$ Department of Applied Mathematics, University of Crete, and IACM/FORTH, Heraklion 70013, Greece (tsogka@ tem.uoc.gr, spapadem@tem.uoc.gr).

${ }^{\ddagger}$ Archimedes Center for Modeling, Analysis \& Computation (ACMAC), Department of Applied Mathematics, University of Crete, and IACM/FORTH, Heraklion 70013, Greece (dmits@tem.uoc.gr).
} 
of our waveguide) and use appropriate imaging functionals, which have the property that their values, when they are computed and graphically displayed in the search domain, exhibit peaks that indicate the presence of the scatterer. Examples of such imaging functionals include the Kirchhoff migration functional (see $[3,5]$ ) and the matched field functional (see [16]).

Another question that often arises in imaging of extended reflectors is whether we can create an image which focuses on specific parts of the scatterer. A way to achieve this is by a selective imaging technique called the subspace projection method [4]. This method is based on the singular value decomposition (SVD) (see, for example, [10, sect. 2.5]) of the array response matrix, which helps us to create a filtered version of it which, in turn, will be used for imaging purposes. This technique was employed in [4] to image extended scatterers embedded in a homogeneous medium, while in [7] the authors considered selective imaging in clutter, i.e., propagation media with inhomogeneities that are unknown, cannot be estimated in detail, and are modeled as random processes.

The concept of selective imaging of extended scatterers has been motivated by the concept of selective focusing, which concerns the case where there are multiple point (or small) scatterers in the medium and, essentially, allows one to distinguish specific scatterers by creating images that focus separately in each of them. In this direction, the work of Prada and Fink [20] has been very influential. They have introduced the so-called DORT method (DORT is an acronym for "decomposition of the time reversal operator" in French), which uses the SVD of the time reversal operator to focus selectively on scattering obstacles. The experimental results obtained in [20] show that for small scatterers, the number of nonzero (or significant) singular values of the response matrix is exactly the number of obstacles contained in the medium. Furthermore, the use of the corresponding singular vectors as an incident field results in selective focusing on the scatterers, provided that pairwise they are separated by a sufficiently large distance. Related works include [17, 19]. When the scatterers are clustered together, the one-to-one correspondence between the singular vectors of the response matrix and the scatterers does not hold any longer, and, as a consequence, selective focusing cannot be achieved with DORT. This issue was addressed in [6], where selective focusing was achieved by using as illumination an optimal convex combination of the leading singular vectors across the bandwidth. To the best of our knowledge, the first rigorous mathematical justification of DORT has been given in [13], where the propagation medium is the free space $\mathbb{R}^{3}$. For the analysis of DORT in a waveguide environment we refer the reader to the work of Pinçon and Ramdani [18].

The main goal of the current paper is to propose and analyze a selective imaging method of extended reflectors in waveguides. Moreover, we want to investigate whether, and how, the number of "significant" (nonzero) singular values of the array response matrix is related to the size of the reflector. Relevant works in the same spirit, but for the free space case, include [26], where an analysis of the response matrix for extended reflectors is presented, and [4], where the subspace projection method has been analyzed and a relation between the number of significant singular values and the size of the reflector has been derived.

This paper is organized as follows. In section 2, we formulate the problem, present the basic features of wave propagation in waveguides, and introduce the relevant notation and terminology. In section 3 we present the imaging functionals that will be used throughout this paper. These are the Kirchhoff migration functional and an alternative imaging functional, 


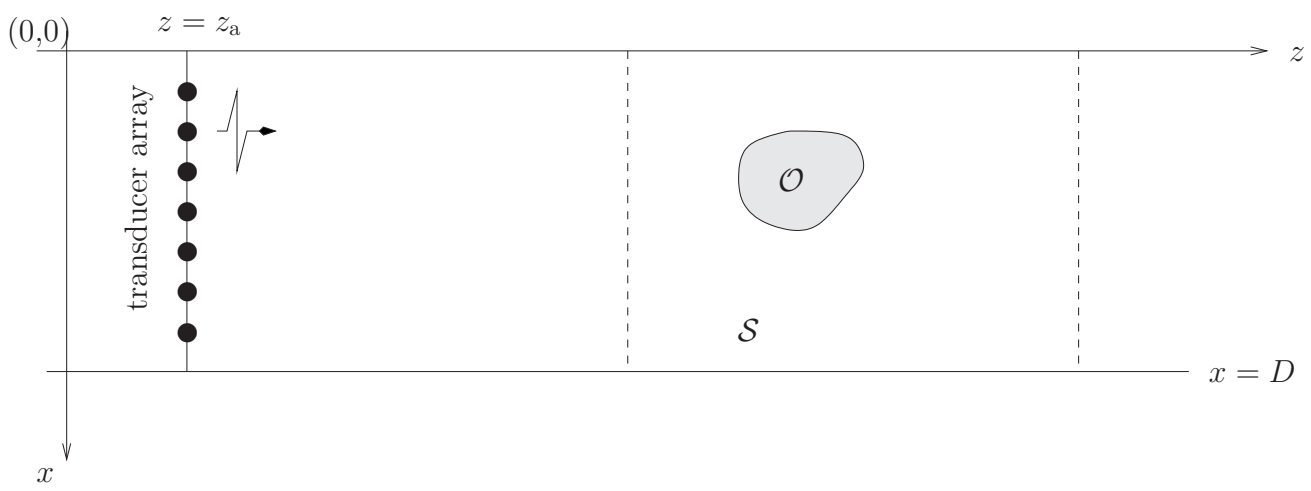

Figure 1. Schematic representation of our waveguide problem and the active array imaging setup.

based on a weighted projection of the response matrix on the propagating modes. In section 4 , we present numerical results of selective imaging for various shapes of extended reflectors. Section 5 is devoted to the theoretical analysis of the imaging method for a simplified model problem where the scatterer is a crack, i.e., a vertical one-dimensional perfect reflector. For this model problem, we derive a relation between the number of significant singular values of the array response matrix and the size of our scatterer. Specifically, we show that the number of significant singular values equals the size of the scatterer divided by the array resolution. This result, which is physically intuitive as pointed out in $[14,22,23]$, was first derived analytically in [5] for a crack in free space and then in [4] for the more general case of extended reflectors in the so-called Fraunhofer diffraction regime. Here, we derive it analytically for a crack embedded in a waveguide and observe it numerically for more general extended reflectors in waveguides. In the case of the crack, we also derive analytic expressions for the singular vectors of the response matrix, and this allows us to study in detail the behavior of our selective imaging functional. We offer some concluding remarks in section 6 .

2. Formulation of the problem. We consider the problem of detecting and imaging extended scatterers submerged in the sea using acoustic waves. More precisely, we model a marine environment by an infinite two-dimensional waveguide $\mathbb{R} \times(0, D)$ in Cartesian coordinates $(z, x)$, where $z$ denotes the range variable and $x$ the depth (cross-range) variable taken to be positive downward. Throughout this paper, vectors in $\mathbb{R}^{n}$ are denoted by boldface characters, while vectors in $\mathbb{R} \times(0, D)$ are denoted by boldface characters with an overscript arrow. Our waveguide consists of a single water layer with constant density and constant sound speed $c_{0}$. A single extended scatterer denoted by $\mathcal{O}$ is submerged in the water layer; see Figure 1. The term "extended" indicates that the typical size of the scatterer is comparable to the wavelength.

We assume that the total acoustic pressure field $p^{\text {tot }}(t, \overrightarrow{\boldsymbol{x}})$ satisfies the wave equation

$$
\Delta p^{\mathrm{tot}}(t, \overrightarrow{\boldsymbol{x}})-\frac{1}{c_{0}^{2}} \frac{\partial^{2} p^{\mathrm{tot}}(t, \overrightarrow{\boldsymbol{x}})}{\partial t^{2}}=f(t, \overrightarrow{\boldsymbol{x}}),
$$

where the source term is of the form $f(t, \overrightarrow{\boldsymbol{x}})=-\exp (-\mathrm{i} \omega t) \delta(t) \delta\left(\overrightarrow{\boldsymbol{x}}-\overrightarrow{\boldsymbol{x}}_{\mathrm{s}}\right)$, modeling a point-like source with time harmonic dependence located at $\overrightarrow{\boldsymbol{x}}_{\mathrm{s}}$. The scatterer is assumed to be sound- 
hard; hence a homogeneous Neumann condition is posed on its boundary $\partial \mathcal{O}$. Equation (2.1) is supplemented with pressure release boundary conditions on the surface and the seafloor, and we also assume that $p^{\text {tot }}(t, \overrightarrow{\boldsymbol{x}})=0$ for $t \leq 0$, expressing that the medium is quiescent before emission. Taking the Fourier transform

$$
\widehat{p}^{\mathrm{tot}}(\omega, \overrightarrow{\boldsymbol{x}})=\int \mathrm{e}^{\mathrm{i} \omega t} p^{\mathrm{tot}}(t, \overrightarrow{\boldsymbol{x}}) d t
$$

we obtain from (2.1) the Helmholtz equation

$$
-\Delta \widehat{p}^{\mathrm{tot}}(\omega, \overrightarrow{\boldsymbol{x}})-k^{2} \widehat{p}^{\mathrm{tot}}(\omega, \overrightarrow{\boldsymbol{x}})=\delta\left(\overrightarrow{\boldsymbol{x}}-\overrightarrow{\boldsymbol{x}}_{\mathrm{s}}\right),
$$

where $k=\omega / c_{0}$ is the real wavenumber, $\omega$ is the angular frequency, and $\lambda=2 \pi / k$ is the wavelength.

Now let $\left\{\mu_{n}, X_{n}\right\}_{n=1,2, \ldots}$ denote the eigenvalues and corresponding orthonormal eigenfunctions of the two-point vertical eigenvalue problem

$$
X^{\prime \prime}(x)+\mu X(x)=0, x \in(0, D), \quad \text { and } \quad X(0)=X(D)=0,
$$

i.e.,

$$
\mu_{n}=(n \pi / D)^{2}, \quad X_{n}(x)=\sqrt{2 / D} \sin \left(\sqrt{\mu_{n}} x\right), n=1,2, \ldots
$$

The family of eigenfunctions $\left\{X_{n}\right\}_{n=1,2, \ldots}$ forms an orthonormal basis of $L^{2}[0, D]$. We assume that for each frequency there exists an index $M$ such that

$$
\mu_{M}<k<\mu_{M+1} .
$$

In other words, the wavenumber does not coincide with any of the mode cutoff frequencies, and the first $M$ eigenvalues correspond to the propagating modes, while the rest correspond to the evanescent modes. Let us also denote the horizontal wavenumbers by

$$
\beta_{n}= \begin{cases}\sqrt{k^{2}-\mu_{n}}, & 1 \leq n \leq M \\ \mathrm{i} \sqrt{\mu_{n}-k^{2}}, & n \geq M+1 .\end{cases}
$$

Moreover, let $\widehat{G}\left(\overrightarrow{\boldsymbol{x}}, \overrightarrow{\boldsymbol{x}}_{\mathrm{s}}\right)$ be the outgoing Green's function of the Helmholtz operator $-\Delta$. $-k^{2}$, evaluated at $\overrightarrow{\boldsymbol{x}}=(z, x) \in \mathbb{R} \times(0, D)$ due to a point source located at $\overrightarrow{\boldsymbol{x}}_{\mathrm{s}}=\left(z_{\mathrm{s}}, x_{\mathrm{s}}\right)$. (In the underwater acoustics community, a point source in plane geometry is usually referred to as a line source [15].) Then it is well known (see, e.g., $[15,18]$ ) that $\widehat{G}$ admits the following normal mode representation:

$$
\widehat{G}\left(\overrightarrow{\boldsymbol{x}}, \overrightarrow{\boldsymbol{x}}_{\mathrm{s}}\right)=\frac{\mathrm{i}}{2} \sum_{n=1}^{\infty} \frac{1}{\beta_{n}} \mathrm{e}^{\mathrm{i} \beta_{n}\left|z-z_{\mathrm{s}}\right|} X_{n}(x) X_{n}\left(x_{\mathrm{s}}\right),
$$

where $\left\{\mu_{n}, X_{n}\right\}$ and $\beta_{n}$ are defined in (2.3) and (2.4), respectively. 
2.1. Array imaging setup. We consider a one-dimensional vertical active array spanning the whole depth of the waveguide and located at range $z=z_{\mathrm{a}} \geq 0$; see Figure 1 . The array consists of $N$ transducers which act as both sources and receivers. We assume that they are closely spaced and uniformly distributed on the array with an interelement array distance $h=D /(N+1) ; h$ is usually called the array pitch.

Also let $\widehat{\Pi}(\omega)$ denote the $N \times N$ complex array response matrix in the frequency domain, whose $(r, s)$ entry is defined as the Fourier transform of the time traces recorded at the $r$ th transducer due to a $\delta$-function impulse generated by the $s$ th transducer for a given frequency $\omega$. In what follows, the data that we are going to use for imaging is the array response matrix for the scattered field in the frequency domain, created by subtracting the array response matrix for the incident field from the corresponding one for the total field.

3. Imaging. Let us first define the search domain $\mathcal{S}$ as a bounded subdomain of our waveguide that may contain a scatterer (see Figure 1). $\mathcal{S}$ is discretized, using a rectangular grid, and $\overrightarrow{\boldsymbol{y}}^{\mathrm{s}}=\left(z^{\mathrm{s}}, x^{\mathrm{S}}\right)$ denotes an arbitrary node in $\mathcal{S}$. We are interested in creating an image of the search domain $\mathcal{S}$. A classical imaging method is Kirchhoff migration $(K M)[3,5]$, defined by

$$
\mathcal{I}^{\mathrm{KM}}\left(\overrightarrow{\boldsymbol{y}}^{\mathrm{s}}, \omega\right)=\sum_{r=1}^{N} \overline{\widehat{G}\left(\overrightarrow{\boldsymbol{x}}_{r}, \overrightarrow{\boldsymbol{y}}^{\mathrm{s}}, \omega\right)} \sum_{s=1}^{N} \widehat{\Pi}\left(\overrightarrow{\boldsymbol{x}}_{r}, \overrightarrow{\boldsymbol{x}}_{s}, \omega\right) \overline{\widehat{G}\left(\overrightarrow{\boldsymbol{x}}_{s}, \overrightarrow{\boldsymbol{y}}^{\mathrm{s}}, \omega\right)}
$$

for a single frequency $\omega$ and $\overrightarrow{\boldsymbol{y}}^{\mathrm{s}} \in \mathcal{S}$. Here the bars denote complex conjugation. KM consists in backpropagating the signals from each receiver $\overrightarrow{\boldsymbol{x}}_{r}$ to a point $\overrightarrow{\boldsymbol{y}}^{\mathrm{s}}$ in the search domain and then back to the source $\overrightarrow{\boldsymbol{x}}_{s}$. The image is the sum of the backpropagated signals for all sources and receivers.

In (3.1) we have defined the imaging functional for a single frequency $\omega$. When we have multifrequency data, we can compute instead

$$
\mathcal{I}^{\mathrm{KM}}\left(\overrightarrow{\boldsymbol{y}}^{\mathrm{s}}\right)=\left|\sum_{\omega} \mathcal{I}^{\mathrm{KM}}\left(\overrightarrow{\boldsymbol{y}}^{\mathrm{s}}, \omega\right)\right| .
$$

KM is widely used in seismic imaging and exploration geophysics; in these applications the arrays and the bandwidth are typically very large. This is a setup in which perfect imaging resolution can be achieved. Indeed, in this case the ideal point spread function (i.e., the image of a point scatterer), which is a Dirac distribution, can be obtained, at least asymptotically, as the array aperture and the bandwidth tend to infinity (cf. [3]). To be more precise, this result is obtained for a slight modification of (3.1) that uses a weighing factor that takes into account the source-receiver geometry.

3.1. Selective imaging. In selective imaging we are interested in reconstructing specific parts of the reflector such as, for example, its boundary. A way to achieve this is by means of the subspace projection method [4], which is based on the SVD of the $N \times N$ array response matrix $\widehat{\Pi}(\omega)$ in the frequency domain. As remarked in [4], the SVD of $\widehat{\Pi}(\omega)$ may serve as a filter which enables us to identify reflections emanating from the edges of the scatterer. Such reflections are typically weaker than, and therefore masked by, those coming from the body of 
the scatterer. The SVD of $\widehat{\Pi}(\omega)$ is a factorization of the form (see, for example, [10, sect. 2.5])

$$
\widehat{\Pi}(\omega)=U(\omega) \Sigma(\omega) V^{*}(\omega)
$$

where $\Sigma$ is a diagonal matrix containing the singular values $\sigma_{i}$ of $\widehat{\Pi}(\omega)$ in descending order, and $U, V$ are unitary matrices containing the left and right singular vectors, respectively. For the rest of this section, we will occasionally omit the $\omega$ 's for the sake of notational convenience.

Now, let us write the SVD of $\widehat{\Pi}(\omega)$ as a sum of the form

$$
\widehat{\Pi}(\omega)=\sum_{i=1}^{\rho} \sigma_{i} U_{i} V_{i}^{*},
$$

where $\rho=\operatorname{rank}(\widehat{\Pi}(\omega))$, so that $\sigma_{1} \geq \cdots \geq \sigma_{\rho}>\sigma_{\rho+1}=\cdots=\sigma_{N}=0$, and $U_{i}, V_{i}$ are the left and right singular vectors, respectively. Then a filtered version of the response matrix may be written in the form

$$
D[\widehat{\Pi}(\omega)]=\sum_{i=1}^{\rho} d_{i} \sigma_{i} U_{i} V_{i}^{*},
$$

where the coefficients $d_{i}$ are called the filter weights. We will simply consider $d_{i} \in\{0,1\}$, which amounts to saying that if $d_{i}=1$, then the $i$ th singular vector is taken into account in the filtered version of the response matrix, while if $d_{i}=0$, it is not.

We will assume in the following that the scatterer and the search domain are located far enough from the array to allow us to retain only the propagating modes in (2.5). Then, substituting $\widehat{G}$, given by (2.5), into (3.1), we may write the KM functional in the form

$$
\begin{aligned}
\mathcal{I}^{\mathrm{KM}}\left(\overrightarrow{\boldsymbol{y}}^{\mathrm{s}}, \omega\right)= & -\frac{1}{4} \sum_{s, r=1}^{N} \widehat{\Pi}\left(\overrightarrow{\boldsymbol{x}}_{r}, \overrightarrow{\boldsymbol{x}}_{s}, \omega\right) \\
& \times \sum_{m, n=1}^{M} \frac{\mathrm{e}^{-\mathrm{i}\left(\beta_{m}+\beta_{n}\right)\left|z_{\mathrm{a}}-z^{\mathrm{s}}\right|}}{\beta_{m} \beta_{n}} X_{m}\left(x_{s}\right) X_{m}\left(x^{\mathrm{s}}\right) X_{n}\left(x_{r}\right) X_{n}\left(x^{\mathrm{s}}\right) .
\end{aligned}
$$

Let us also define the functional

$$
\begin{aligned}
\mathcal{I}^{\mathrm{KM}, \mathrm{f}}\left(\overrightarrow{\boldsymbol{y}}^{\mathrm{s}}, \omega\right)= & -\frac{1}{4} \sum_{s, r=1}^{N}(D[\widehat{\Pi}(\omega)])_{r s} \\
& \times \sum_{m, n=1}^{M} \frac{\mathrm{e}^{-\mathrm{i}\left(\beta_{m}+\beta_{n}\right)\left|z_{\mathrm{a}}-z^{\mathrm{s}}\right|}}{\beta_{m} \beta_{n}} X_{m}\left(x_{s}\right) X_{m}\left(x^{\mathrm{s}}\right) X_{n}\left(x_{r}\right) X_{n}\left(x^{\mathrm{s}}\right),
\end{aligned}
$$

derived by replacing the full response matrix by its filtered version. For multifrequency data, we define

$$
\mathcal{I}^{\mathrm{KM}, \mathrm{f}}\left(\overrightarrow{\boldsymbol{y}}^{\mathrm{s}}\right)=\left|\sum_{\omega} \mathcal{I}^{\mathrm{KM}, \mathrm{f}}\left(\overrightarrow{\boldsymbol{y}}^{\mathrm{s}}, \omega\right)\right|
$$


Finally, we introduce the functionals

$$
\begin{aligned}
\mathcal{I}_{J}^{\mathrm{KM}}\left(\overrightarrow{\boldsymbol{y}}^{\mathrm{s}}, \omega\right)= & -\frac{1}{4} \sum_{s, r=1}^{N}\left(\sigma_{J}(\omega) U_{J}(\omega) V_{J}^{*}(\omega)\right)_{r s} \\
& \times \sum_{m, n=1}^{M} \frac{\mathrm{e}^{-\mathrm{i}\left(\beta_{m}+\beta_{n}\right)\left|z_{\mathrm{a}}-z^{\mathrm{s}}\right|}}{\beta_{m} \beta_{n}} X_{m}\left(x_{s}\right) X_{m}\left(x^{\mathrm{s}}\right) X_{n}\left(x_{r}\right) X_{n}\left(x^{\mathrm{s}}\right)
\end{aligned}
$$

and

$$
\mathcal{I}_{J}^{\mathrm{KM}}\left(\overrightarrow{\boldsymbol{y}}^{\mathrm{s}}\right)=\left|\sum_{\omega} \mathcal{I}_{J}^{\mathrm{KM}}\left(\overrightarrow{\boldsymbol{y}}^{\mathrm{s}}, \omega\right)\right|
$$

which are derived from (3.4) and (3.5), respectively, when $d_{J}=1$ and $d_{i}=0$ for all $i \neq J$, in other words, when we consider projection onto the single $J$ th singular vector of the response matrix.

3.2. Modal projection and selective imaging. For an array spanning the whole waveguide depth with an array pitch $h$ small enough, we can approximate the double sum in (3.3) by a double integral over $[0, D]$ to get

$$
\begin{aligned}
\mathcal{I}^{\mathrm{KM}}\left(\overrightarrow{\boldsymbol{y}}^{\mathrm{s}}, \omega\right) \approx & -\frac{1}{4 h^{2}} \int_{0}^{D} \mathrm{~d} x_{s} \int_{0}^{D} \mathrm{~d} x_{r} \widehat{\Pi}\left(\overrightarrow{\boldsymbol{x}}_{s}, \overrightarrow{\boldsymbol{x}}_{r}, \omega\right) \\
& \times \sum_{m, n=1}^{M} \frac{\mathrm{e}^{-\mathrm{i}\left(\beta_{m}+\beta_{n}\right)\left|z_{\mathrm{a}}-z^{\mathrm{s}}\right|}}{\beta_{m} \beta_{n}} X_{m}\left(x_{s}\right) X_{m}\left(x^{\mathrm{s}}\right) X_{n}\left(x_{r}\right) X_{n}\left(x^{\mathrm{s}}\right) \\
= & -\frac{1}{4 h^{2}} \sum_{m, n=1}^{M} \frac{\mathrm{e}^{-\mathrm{i}\left(\beta_{m}+\beta_{n}\right)\left|z_{\mathrm{a}}-z^{\mathrm{s}}\right|}}{\beta_{m} \beta_{n}} X_{m}\left(x^{\mathrm{s}}\right) X_{n}\left(x^{\mathrm{s}}\right) \\
& \times \int_{0}^{D} \int_{0}^{D} \widehat{\Pi}\left(\overrightarrow{\boldsymbol{x}}_{s}, \overrightarrow{\boldsymbol{x}}_{r}, \omega\right) X_{m}\left(x_{s}\right) X_{n}\left(x_{r}\right) \mathrm{d} x_{r} \mathrm{~d} x_{s} .
\end{aligned}
$$

Now, let us introduce an $M \times M$ matrix $\widehat{\mathbb{Q}}(\omega)$ with entries

$$
\widehat{\mathbb{Q}}_{m n}(\omega)=\int_{0}^{D} \mathrm{~d} x_{s} \int_{0}^{D} \mathrm{~d} x_{r} \widehat{\Pi}\left(\overrightarrow{\boldsymbol{x}}_{s}, \overrightarrow{\boldsymbol{x}}_{r}, \omega\right) X_{m}\left(x_{s}\right) X_{n}\left(x_{r}\right)
$$

for $m, n=1,2, \ldots, M$.

In view of (3.9), (3.8) may be equivalently written as

$$
\mathcal{I}^{\mathrm{KM}}\left(\overrightarrow{\boldsymbol{y}}^{\mathrm{s}}, \omega\right) \approx-\frac{1}{4 h^{2}} \sum_{m, n} \frac{\mathrm{e}^{-\mathrm{i}\left(\beta_{m}+\beta_{n}\right)\left|z_{\mathrm{a}}-z^{\mathrm{s}}\right|}}{\beta_{m} \beta_{n}} X_{n}\left(x^{\mathrm{s}}\right) X_{m}\left(x^{\mathrm{s}}\right) \widehat{\mathbb{Q}}_{m n}(\omega) .
$$

Instead of using (3.10) we propose using the following imaging functional:

$$
\widetilde{\mathcal{I}}^{\mathrm{KM}}\left(\overrightarrow{\boldsymbol{y}}^{\mathrm{s}}, \omega\right)=-\frac{1}{4 h^{2}} \sum_{m, n=1}^{M} \mathrm{e}^{-\mathrm{i}\left(\beta_{m}+\beta_{n}\right)\left|z_{\mathrm{a}}-z^{\mathrm{s}}\right|} X_{n}\left(x^{\mathrm{s}}\right) X_{m}\left(x^{\mathrm{s}}\right) \widehat{\mathbb{P}}_{m n}(\omega),
$$


where the $M \times M$ matrix $\widehat{\mathbb{P}}$ is defined as

$$
\widehat{\mathbb{P}}_{m n}(\omega)=\beta_{m} \beta_{n} \widehat{\mathbb{Q}}_{m n}, \quad m, n=1, \ldots, M .
$$

Note that $\widehat{\mathbb{P}}_{m n}(\omega)$ is a weighted modal projection of the array response matrix. Our numerical results indicate (see section 4 ) that (3.11) is a robust selective imaging functional, while this is not the case for (3.10). Our choice of imaging with (3.11) is also justified by the theoretical analysis carried out in section 5 for the case of a simple scatterer geometry.

For multifrequency data, we define

$$
\widetilde{\mathcal{I}}^{\mathrm{KM}}\left(\overrightarrow{\boldsymbol{y}}^{\mathrm{s}}\right)=\left|\sum_{\omega} \widetilde{\mathcal{I}}^{\mathrm{KM}}\left(\overrightarrow{\boldsymbol{y}}^{\mathrm{s}}, \omega\right)\right| \cdot
$$

We also introduce functionals for selective imaging, as we have done in (3.4)-(3.7), using filtered versions of $\widehat{\mathbb{P}}$. The only thing that changes in this process is the size of the response matrix. Now we are dealing with an $M \times M$ matrix, where $M$ denotes the number of propagating modes. Specifically, for a single frequency $\omega$, we let

$$
\widetilde{\mathcal{I}}^{\mathrm{KM}, \mathrm{f}}\left(\overrightarrow{\boldsymbol{y}}^{\mathrm{s}}, \omega\right)=-\frac{1}{4 h^{2}} \sum_{m, n=1}^{M} \mathrm{e}^{-\mathrm{i}\left(\beta_{n}+\beta_{m}\right)\left|z_{\mathrm{a}}-z^{\mathrm{s}}\right|} X_{n}\left(x^{\mathrm{s}}\right) X_{m}\left(x^{\mathrm{s}}\right)(D[\widehat{\mathbb{P}}(\omega)])_{m n},
$$

while for multifrequency data we define

$$
\widetilde{\mathcal{I}}^{\mathrm{KM}, \mathrm{f}}\left(\overrightarrow{\boldsymbol{y}}^{\mathrm{s}}\right)=\left|\sum_{\omega} \widetilde{\mathcal{I}}^{\mathrm{KM}, \mathrm{f}}\left(\overrightarrow{\boldsymbol{y}}^{\mathrm{s}}, \omega\right)\right| \cdot
$$

Furthermore, we define

$$
\widetilde{\mathcal{I}}_{J}^{\mathrm{KM}}\left(\overrightarrow{\boldsymbol{y}}^{\mathrm{s}}, \omega\right)=-\frac{1}{4 h^{2}} \sum_{m, n=1}^{M} \mathrm{e}^{-\mathrm{i}\left(\beta_{n}+\beta_{m}\right)\left|z_{\mathrm{a}}-z^{\mathrm{s}}\right|} X_{n}\left(x^{\mathrm{s}}\right) X_{m}\left(x^{\mathrm{s}}\right)\left(\sigma_{J}(\omega) U_{J}(\omega) V_{J}^{*}(\omega)\right)_{m n}
$$

and

$$
\widetilde{\mathcal{I}}_{J}^{\mathrm{KM}}\left(\overrightarrow{\boldsymbol{y}}^{\mathrm{s}}\right)=\left|\sum_{\omega} \widetilde{\mathcal{I}}_{J}^{\mathrm{KM}}\left(\overrightarrow{\boldsymbol{y}}^{\mathrm{s}}, \omega\right)\right|,
$$

where, by slightly abusing the notation, we denote by $\sigma_{J}(\omega) U_{J}(\omega) V_{J}^{*}(\omega)$ the projection onto the $J$ th singular vector of $\widehat{\mathbb{P}}(\omega)$.

Remark 1. One of the main assumptions that is essential for the construction of the proposed imaging functional, and for the subsequent analysis in section 5 , is that the array spans the whole waveguide, a hypothesis that is probably not realistic in applications. We are currently investigating the generalization of this approach to the case of partial array aperture. In this case the analysis does not seem at all straightforward as, for example, key properties such as the orthonormality of the vertical eigenfunctions $X_{n}$ along the array fail to hold. 


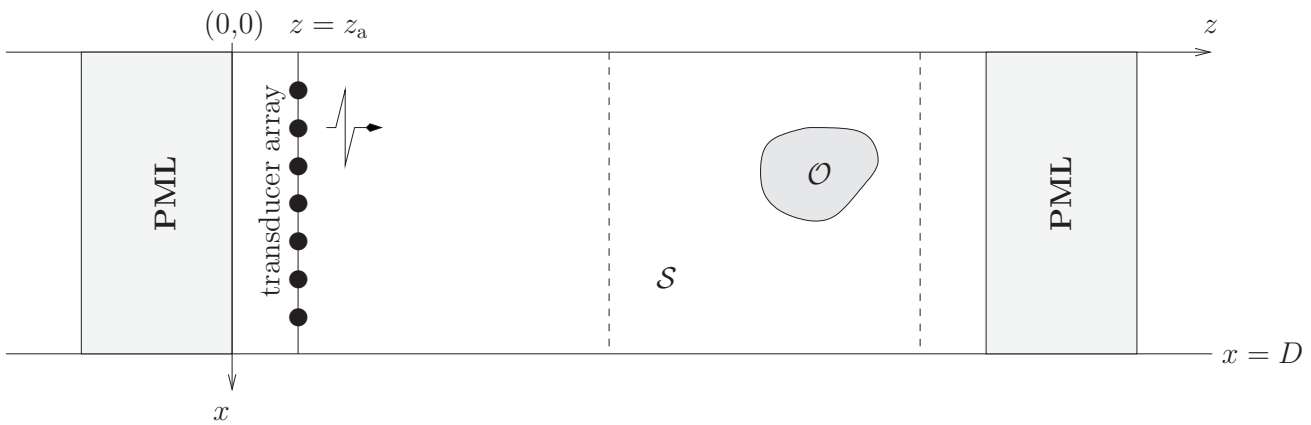

Figure 2. Schematic representation of a waveguide truncated near and far from the source with two perfectly matched layers (PMLs).

4. Numerical experiments. In this section we present numerical experiments for scatterers of various shapes and sizes. In order to construct the array response matrix, which is necessary for evaluating the imaging functionals, we solve numerically the wave equation problem (2.1). To this end, we use Montjoie (http://montjoie.gforge.inria.fr/), a high-order finite element $\mathrm{C}++$ code developed at INRIA, designed to solve problems arising in wave propagation phenomena, such as acoustic, electromagnetic, aeroacoustic, and elastodynamic problems.

We consider a waveguide with depth equal to $D=200 \mathrm{~m}$ and sound speed $c_{0}=1500 \mathrm{~m} / \mathrm{s}$. The vertical array is placed at $z_{\mathrm{a}}=40 \mathrm{~m}$ and consists of $N=39$ transducers uniformly distributed in the water column with a pitch $h=5 \mathrm{~m}$. Point-like sources are simulated by considering the source term in (2.1) to be of the form $f(t, \overrightarrow{\boldsymbol{x}})=h(t) g\left(\overrightarrow{\boldsymbol{x}} ; \overrightarrow{\boldsymbol{x}}_{s}\right)$. Here $h(t)$ is a Ricker function of time, given by

$$
\left.h(t)=\sqrt{2} f_{0}\left[1-4 \pi^{2} f_{0}^{2}\left(t-t_{c}\right)\right)^{2}\right] \exp \left\{-\left[\sqrt{2} \pi f_{0}\left(t-t_{c}\right)\right]^{2}\right\},
$$

where $f_{0}$ is the central frequency of the pulse and $t_{c}$ is the time at which the source attains its maximum. In the numerical results that follow, $f_{0}=75 \mathrm{~Hz}, t_{c}=0.01 \mathrm{~s}$, and the final computation time is taken as $T=4 \mathrm{~s}$. The function $g\left(\overrightarrow{\boldsymbol{x}} ; \overrightarrow{\boldsymbol{x}}_{s}\right)$ is a Gaussian, given by $g\left(\overrightarrow{\boldsymbol{x}} ; \overrightarrow{\boldsymbol{x}}_{s}\right)=$ $\sqrt{2 \pi / \alpha} \exp \left(-\alpha\left|\overrightarrow{\boldsymbol{x}}-\overrightarrow{\boldsymbol{x}}_{s}\right|^{2}\right)$, where $\alpha=\ln \left(10^{6}\right) / r^{2} ; r$ determines the support of the Gaussian and is taken as $10 \mathrm{~m}$.

The originally infinite (in the $z$-direction) domain is truncated by introducing two perfectly matched layers (PMLs) [2, 9]; see Figure 2. The one near the source is confined in range $[-100,0]$, while the other, far from the source, is confined in [500,600] (all distances are in meters). We have checked that the width of $100 \mathrm{~m}$ for the PMLs was enough to absorb the waves efficiently. The resulting computational domain is discretized with quadrangles on which the usual basis functions of the $\mathbb{Q}_{n}$ family $\left(\mathbb{Q}_{n}=\operatorname{span}\left\{x^{\ell} y^{m}, 0 \leq \ell, m \leq n\right\}\right)$ for $n=8$ are used. Numerical quadrature is based on Gauss-Lobatto rules, and time discretization employs a fourth-order leapfrog scheme.

In all cases the frequencies that are used are close to a central reference frequency $f_{0}=$ $75 \mathrm{~Hz}$, for which the corresponding wavelength is equal to $\lambda_{0}=20 \mathrm{~m}$. In particular, we consider frequencies ranging from 70.5 to $79.5 \mathrm{~Hz}$ with an increment of $1 \mathrm{~Hz}$. 

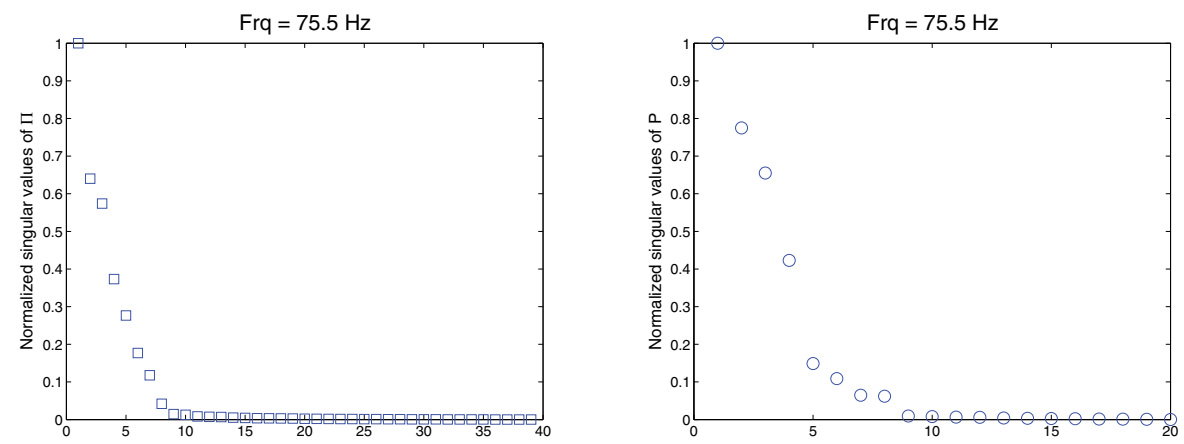

Figure 3. Normalized singular values of $\widehat{\Pi}$ (left subplot) and $\widehat{\mathbb{P}}$ (right subplot) for a circle scatterer with diameter $\delta=40 \mathrm{~m}$.
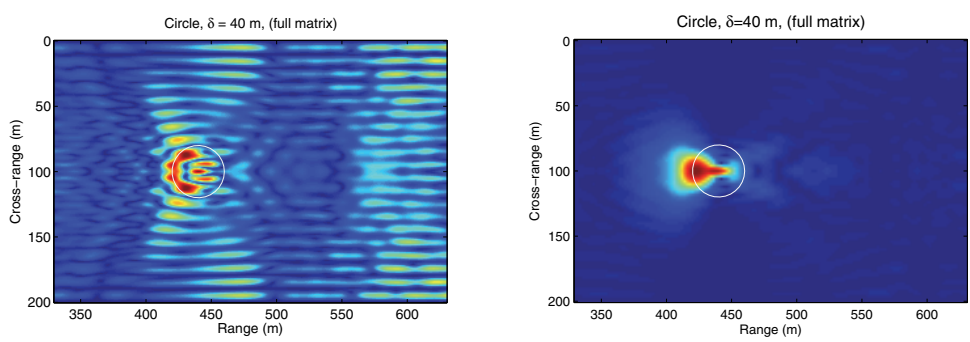

Figure 4. $\mathcal{I}^{\mathrm{KM}}$ (left subplot) versus $\widetilde{\mathcal{I}}^{\mathrm{KM}}$ (right subplot) for a circular scatterer with diameter $\delta=40 \mathrm{~m}$.

In what follows we will examine the performance of the imaging functionals $\mathcal{I}^{\text {KM }}$ and $\widetilde{\mathcal{I}}^{\text {KM }}$. Let us recall the results for selective imaging in free space: It was shown in [4] that information about the edges of a reflector is contained in those singular vectors that correspond to singular values of the response matrix that lie in the intermediate regime between the large ones and zero. In our numerical simulations we observe the same behavior for imaging in waveguides with $\widetilde{\mathcal{I}}^{\mathrm{KM}}$, but this is not the case for $\mathcal{I}^{\mathrm{KM}}$. The behavior of $\widetilde{\mathcal{I}}^{\mathrm{KM}}$ will be explained with the analysis carried out in section 5 .

Test case 1: Circular scatterer with diameter $\delta=40 \mathrm{~m}$. We consider here a circular scatterer with diameter $\delta=2 \lambda_{0}=40 \mathrm{~m}$ centered at $(440,100) \mathrm{m}$. Figure 3 depicts the singular values (normalized with respect to the largest one) of the matrices $\widehat{\Pi}$ and $\widehat{\mathbb{P}}$ for a frequency equal to $75.5 \mathrm{~Hz}$. As expected, only a few of the singular values are nonzero.

In Figure 4 we plot the values of $\mathcal{I}^{\mathrm{KM}}$ and $\widetilde{\mathcal{I}}^{\mathrm{KM}}$ when the full matrices $\widehat{\Pi}$ and $\widehat{\mathbb{P}}$, respectively, are used. In both cases we see that the front part of the circle is recovered, although for $\widetilde{\mathcal{I}}^{\mathrm{KM}}$ the image is supported mainly around the center of the circle at $x=100 \mathrm{~m}$.

In Figure 5 we present the results of selective imaging with $\mathcal{I}_{J}^{\text {KM }}$ and $\widetilde{\mathcal{I}}_{J}^{\text {KM }}$ (see (3.7) and (3.17), respectively) for $J=1,4$, and 5 . For $J=1, \mathcal{I}_{J}^{\mathrm{KM}}$ focuses at the endpoints of the vertical diameter of the circle, while $\widetilde{\mathcal{I}}_{J}^{\mathrm{KM}}$ focuses at the front center of the circle. For $J=4$, $\mathcal{I}_{J}^{\mathrm{KM}}$ seems to focus at the two endpoints of the horizontal diameter of the circle, and $\widetilde{\mathcal{I}}_{J}^{\mathrm{KM}}$ at the endpoints of the vertical diameter. For $J=5$, both $\mathcal{I}_{J}^{\mathrm{KM}}$ and $\widetilde{\mathcal{I}}_{J}^{\mathrm{KM}}$ seem to roughly indicate the boundary of the circle. 

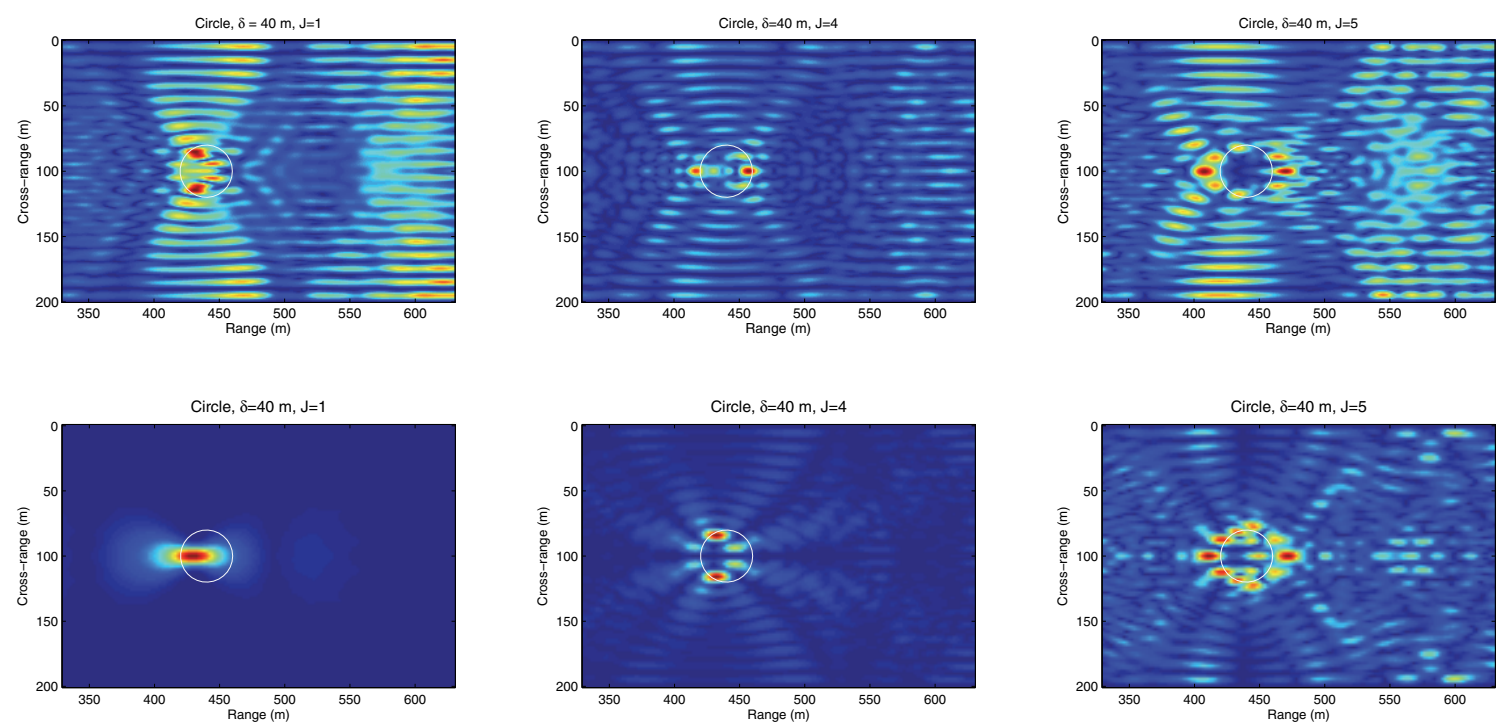

Figure 5. $\mathcal{I}_{J}^{\mathrm{KM}}$ (top row) versus $\widetilde{\mathcal{I}}_{J}^{\mathrm{KM}}$ (bottom row) for a circular scatterer with diameter $\delta=40 \mathrm{~m} . \quad J$ indicates projection onto the $J$ th singular vector. From left to right: $J=1, J=4$, and $J=5$.

As a first comment, note that selective imaging with $\widetilde{\mathcal{I}}^{\mathrm{KM}}$ exhibits the expected behavior (see, e.g., [4]), in the sense that the singular vector corresponding to the largest singular value is associated to an image focused at the center of the object, while the vectors corresponding to the intermediate singular values carry information about the edges.

Test case 2: Circular scatterer with diameter $\delta=20 \mathrm{~m}$ for a single frequency. Here we decrease the diameter of the previous circular scatterer to $\delta=\lambda_{0}=20 \mathrm{~m}$. In Figure 6 we plot the values of $\left|\mathcal{I}_{1}^{\mathrm{KM}}(\omega)\right|$ and $\left|\widetilde{\mathcal{I}}_{1}^{\mathrm{KM}}(\omega)\right|$ (selective imaging with respect to the first singular vector) for single frequencies of $73 \mathrm{~Hz}$ (left column) and $74 \mathrm{~Hz}$ (right column). For both frequencies $\left|\widetilde{\mathcal{I}}_{1}^{\mathrm{KM}}(\omega)\right|$ focuses at the front center of the circle, as opposed to $\left|\mathcal{I}_{1}^{\mathrm{KM}}(\omega)\right|$ which focuses at its edges for $f=73 \mathrm{~Hz}$ but in the center for $f=74 \mathrm{~Hz}$. This kind of lack of robustness has initially motivated us to work with $\widetilde{\mathcal{I}}^{\mathrm{KM}}$ rather than with $\mathcal{I}^{\mathrm{KM}}$.

Test case 3: Rhombus-shaped scatterer with diameter $\delta=40 \mathrm{~m}$. Here we want to assess the performance of $\widetilde{\mathcal{I}}_{J}^{\mathrm{KM}}$ in a more complex test case. Specifically, we consider a rhombus with diameter $\delta=40 \mathrm{~m}$ centered at $(440,100) \mathrm{m}$. In the left subplot of Figure 7 we plot the singular values (normalized with respect to the largest one) of the matrix $\widehat{\mathbb{P}}$ for a frequency equal to $75.5 \mathrm{~Hz}$. Now, the first four singular values are quite close to each other, greater than $80 \%$ of the $\sigma_{1}(\widehat{\mathbb{P}}(\omega))$. The next two are $30 \%-40 \%$ of the largest, the seventh is about $10 \%$, and the rest lie below $5 \%$. In the right subplot we plot the values of $\widetilde{\mathcal{I}}^{\mathrm{KM}}$ using the full matrix $\widehat{\mathbb{P}}$. Figure 8 depicts the values of $\widetilde{\mathcal{I}}_{J}^{\mathrm{KM}}\left(\overrightarrow{\boldsymbol{y}}^{\mathrm{s}}\right)$ for $J=1, \ldots, 6$. Clearly, projection onto the first singular vector leads to focusing at the center of the object. For $J=2,3$, and $4, \widetilde{\mathcal{I}}_{J}^{\mathrm{KM}}$ carries information from the bulk and from the edges, while for $J=5$ and 6 , it focuses at the endpoints of the vertical diameter.

Test case 4: Square scatterer with side length $b=40 \mathrm{~m}$. Now we consider a square scatterer of side length $b=2 \lambda_{0}=40 \mathrm{~m}$, with its center located at $(470,100) \mathrm{m}$. In this test case we examine the behavior of $\widetilde{\mathcal{I}}^{\mathrm{KM}}$ and its filtered version under the influence of instrument 

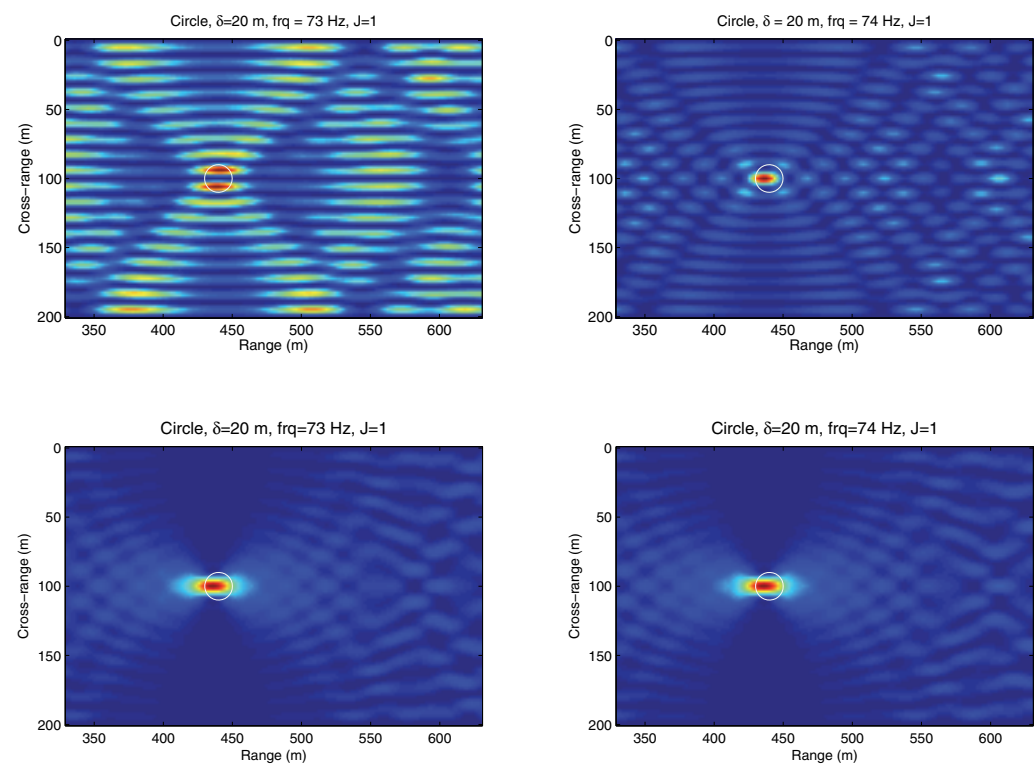

Figure 6. $\left|\mathcal{I}_{1}^{\mathrm{KM}}(\omega)\right|$ (top row) versus $\left|\widetilde{\mathcal{I}}_{1}^{\mathrm{KM}}(\omega)\right|$ (bottom row) for a circle-shaped scatterer with diameter $\delta=20 \mathrm{~m}$ for single frequencies of $73 \mathrm{~Hz}$ (left column) and $74 \mathrm{~Hz}$ (right column).
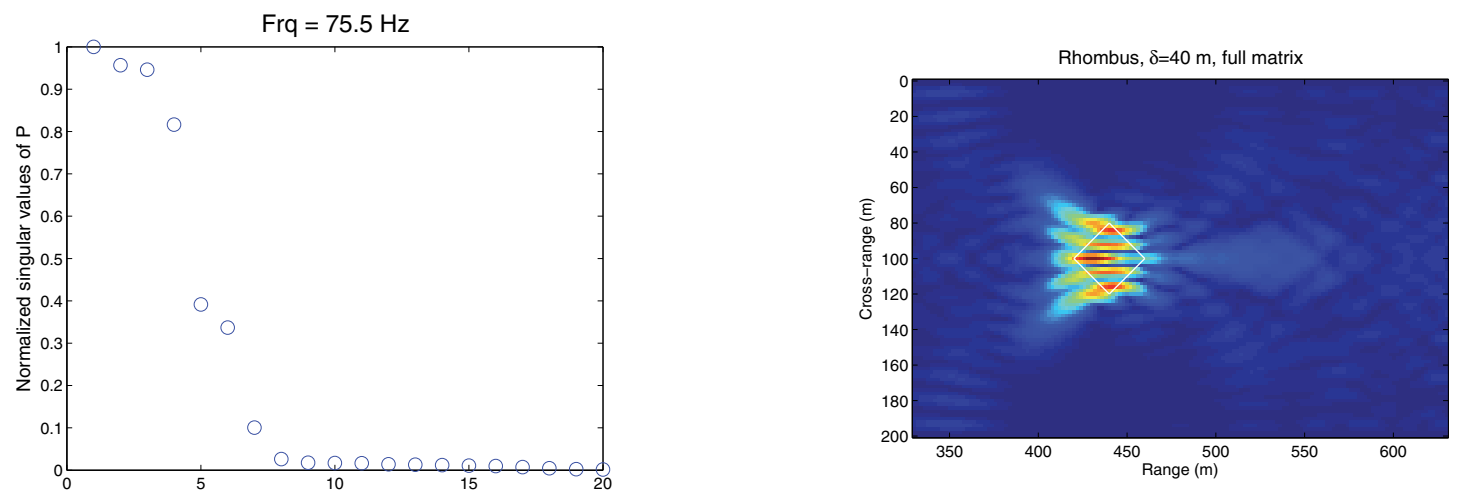

Figure 7. Normalized singular values of $\widehat{\mathbb{P}}$ (left subplot) and $\widetilde{\mathcal{I}}^{\mathrm{KM}}$ for a rhombus-shaped scatterer with diameter $\delta=40 \mathrm{~m}$.

noise. In order to simulate measurement noise we proceed as in [4] and add a noise matrix $W(\omega)$ with zero mean uncorrelated Gaussian distributed entries with variance $\epsilon p_{\text {avg }}$, i.e., $W_{r, s}(\omega) \sim \mathcal{N}\left(0, \epsilon p_{\text {avg }}\right)$. Here the average power received per source, receiver, and frequency is given by

$$
p_{\text {avg }}=\frac{1}{N^{2} N_{\text {freq }}} \sum_{i=1}^{N_{\text {freq }}}\left\|\widehat{\Pi}\left(\omega_{i}\right)\right\|_{\mathrm{F}}^{2},
$$



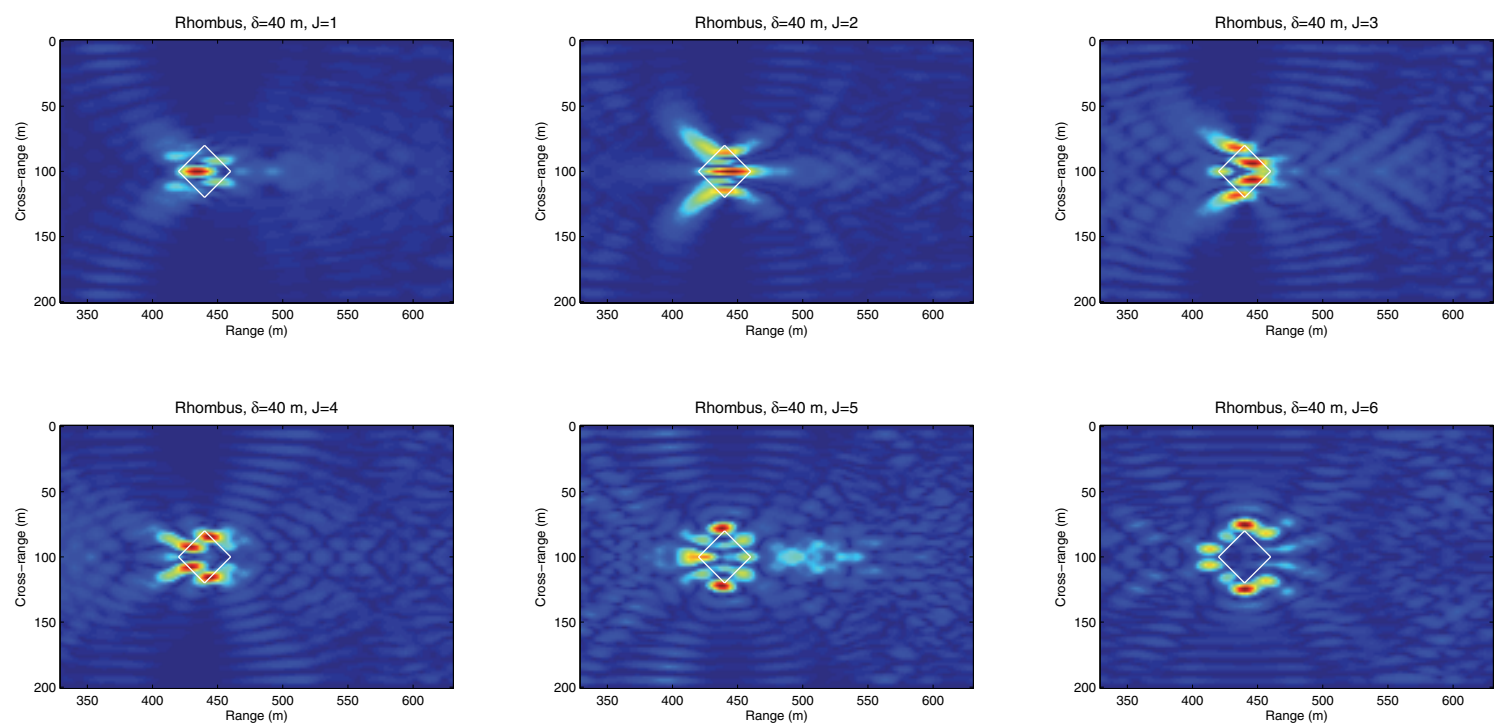

Figure 8. $\tilde{\mathcal{I}}_{J}^{\mathrm{KM}}$ for a rhombus-shaped scatterer with diameter $\delta=40 \mathrm{~m} . \quad J=1, \ldots, 6$ and indicates projection onto the $J$ th singular vector.

where $\|\cdot\|_{F}$ is the Frobenius matrix norm and $N_{\text {freq }}$ the number of frequencies. The expected power of the noise $W\left(\omega_{i}\right)$ over all frequencies, receivers, and sources is

$$
\mathbb{E}\left[\sum_{i=1}^{N_{\text {freq }}}\left\|W\left(\omega_{i}\right)\right\|_{\mathrm{F}}^{2}\right]=\epsilon N^{2} N_{\text {freq }} p_{\text {avg }} .
$$

Since the total power of the signal received over all frequencies, receivers, and sources is $N^{2} N_{\text {freq }} p_{\text {avg }}$, the signal-to-noise ratio (SNR) in dB is $-10 \log _{10} \epsilon$.

As before, we use frequencies ranging from 70.5 to $79.5 \mathrm{~Hz}$ with an increment of $1 \mathrm{~Hz}$. In Figure 9 we plot the singular values of the matrices $\widehat{\Pi}$ and $\widehat{\mathbb{P}}$ for the frequency of $75.5 \mathrm{~Hz}$ (normalized with respect to the largest singular value) in the absence of noise and for SNR $=10,0$, and $-10 \mathrm{~dB}$. Notice that the singular values that are larger than $20 \%$ of the largest singular value in the case of $10 \mathrm{~dB}$ SNR remain close to those corresponding to the unperturbed matrices, while noise has a more profound influence in the singular values of $0 \mathrm{~dB}$ and $-10 \mathrm{~dB}$ SNR.

In Figure 10 we plot the images obtained with $\widetilde{\mathcal{I}}^{\text {KM }}$ without using any selective imaging techniques. In all cases the left side of the scatterer is recreated.

Next, we use filtered versions of the matrix $\widehat{\mathbb{P}}$ that employ more than one singular value. These are chosen as follows [4]:

(a) we normalize the singular values with respect to the largest one,

(b) we determine an interval $[\alpha, \beta] \subset(0,1)$, and

(c) we include in the filtered version of $\widetilde{\mathcal{I}}^{\mathrm{KM}, \mathrm{f}}$ all the singular values that lie in $[\alpha, \beta]$.

Figures $11-12$ depict the values of $\widetilde{\mathcal{I}}^{\mathrm{KM}, \mathrm{f}}$ without noise included and when noise is included with 0 and $-10 \mathrm{~dB}$ SNR. We do not show the images with $10 \mathrm{~dB}$ SNR because they are 

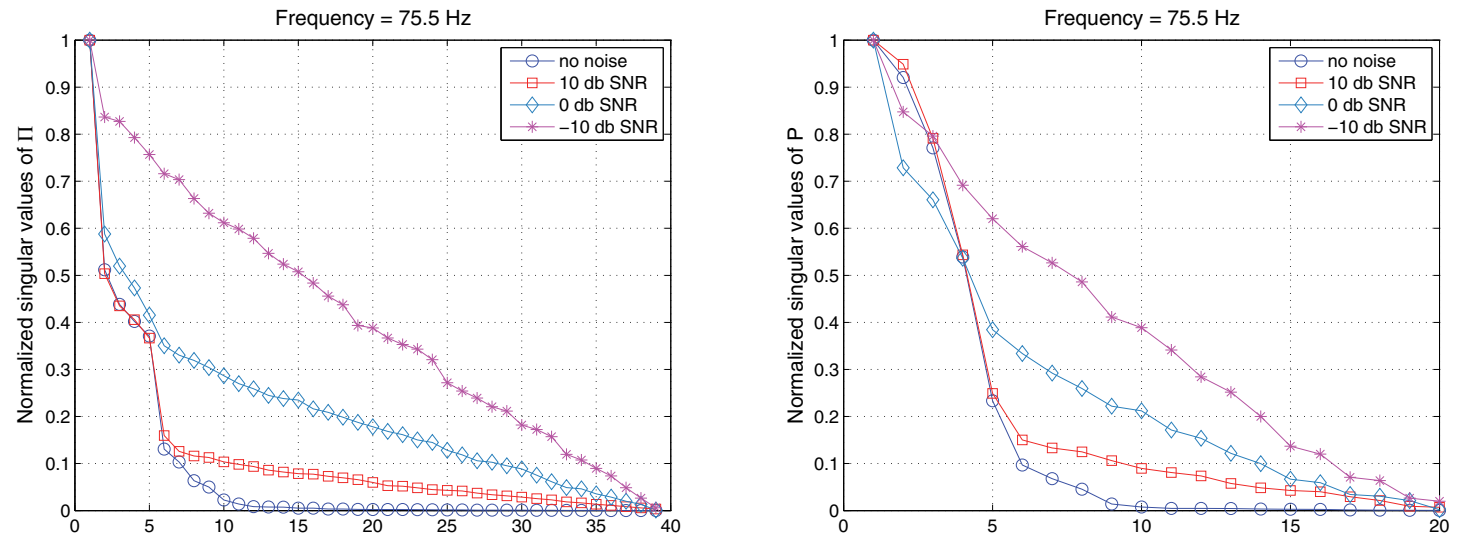

Figure 9. Normalized singular values of $\widehat{\Pi}$ (left subplot) and $\widehat{\mathbb{P}}$ (right subplot) for a square scatterer with side length $b=40 \mathrm{~m}$ in the absence of noise and by adding noise with 10, 0, and $-10 \mathrm{~dB} S N R$.
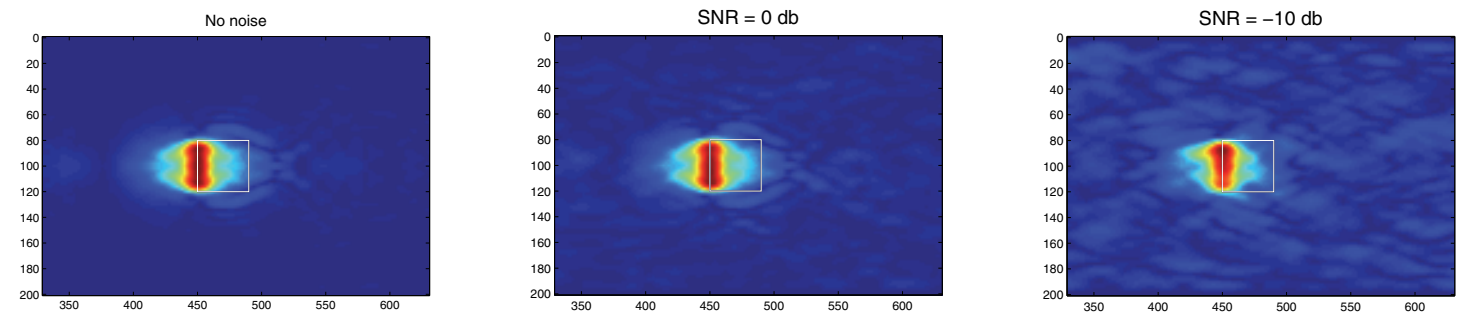

Figure 10. $\widetilde{\mathcal{I}}^{\mathrm{KM}}$ for $b=40 \mathrm{~m}, \mathrm{D}=200 \mathrm{~m}, c_{0}=1500 \mathrm{~m} / \mathrm{s}, f \in[70.5,79.5] \mathrm{Hz}$, and $S N R=\infty, 0,-10 \mathrm{~dB}$.
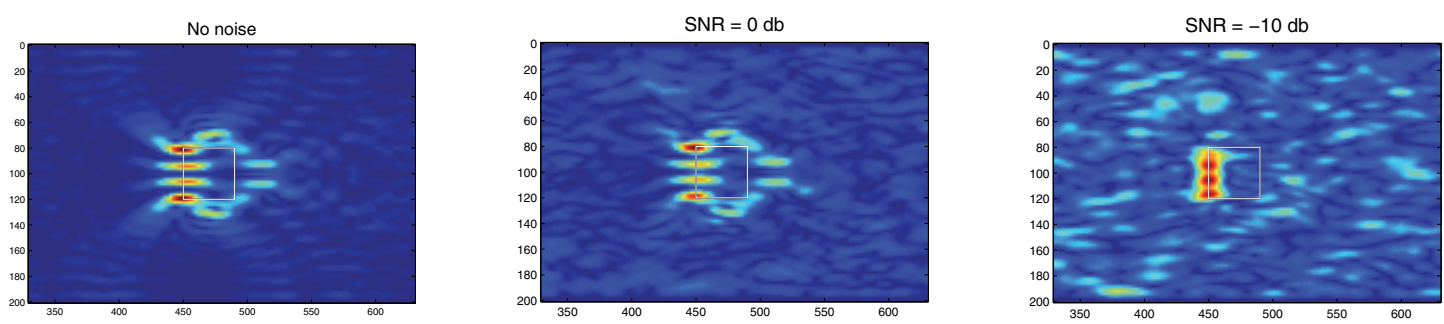

Figure 11. Normalized values of $\tilde{\mathcal{I}}^{\mathrm{KM}, \mathrm{f}}$, for $b=40 \mathrm{~m}, \mathrm{D}=200 \mathrm{~m}, c_{0}=1500 \mathrm{~m} / \mathrm{s}, \mathrm{f} \in[70.5,79.5] \mathrm{Hz}$, and $S N R=\infty, 0,-10 \mathrm{~dB},[\alpha, \beta]=[0.05,0.60]$.

essentially the same as those without noise. Specifically, in Figure 11 we have employed in the computation of $\widetilde{\mathcal{I}}^{\mathrm{KM}, \mathrm{f}}$ the singular values that are $5 \%-60 \%$ of the largest one $([\alpha, \beta]=$ $[0.05,0.60])$, and in Figure 12 we take $[\alpha, \beta]=[0.12,0.74]$. In both cases the results with $0 \mathrm{~dB}$ SNR are qualitatively very similar to those obtained in the absence of noise. Note that even with $-10 \mathrm{~dB}$ SNR, we are still able to recreate the left side of the object, but the effect of the noise is visible in the image. 

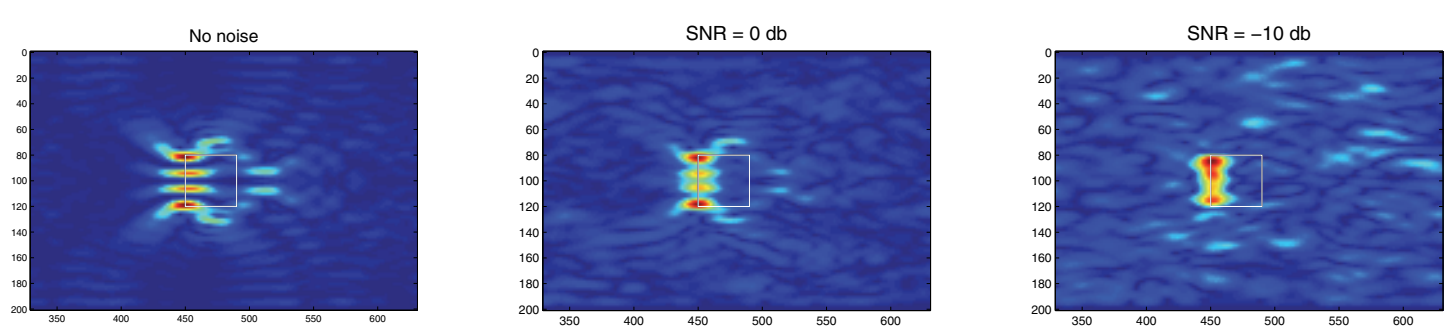

Figure 12. Normalized values of $\widetilde{\mathcal{I}}^{\mathrm{KM}, \mathrm{f}}$, for $b=40 \mathrm{~m}, \mathrm{D}=200 \mathrm{~m}, \mathrm{c}_{0}=1500 \mathrm{~m} / \mathrm{s}, \mathrm{f} \in[70.5,79.5] \mathrm{Hz}$, and $S N R=\infty, 0,-10 \mathrm{~dB},[\alpha, \beta]=[0.12,0.74]$.

5. Analysis of the imaging method. In this section we consider and analyze a simplified model problem that allows us to obtain some explicit expressions of the array response matrix, thus helping us to investigate and understand the phenomena we have observed in the selective imaging approach of the previous sections.

Specifically, we consider the following model problem: In the marine environment described in section 2 we assume that the active array passes through the $x$-axis; i.e., the transducers' coordinates are $\left(0, x_{i}\right), x_{i}=i h, 1 \leq i \leq N$, where $h:=D /(N+1)$ is the pitch. The target, denoted by $\mathcal{T}$, is assumed to be a vertical one-dimensional perfect reflector, i.e., a "crack" of width $b$, located at range $z=L$. The center of the target is denoted by $\overrightarrow{\boldsymbol{y}}^{*}=\left(L, x_{0}\right)$. Let us also denote by $\mathcal{C}$ the vertical section of the waveguide at range $z=L$, i.e., $\mathcal{C}:=\{(L, x): 0 \leq x \leq D\}$. This setup is schematically depicted in Figure 13 and aims at simulating the left side of the square scatterer which we have examined in section 4 . We refer the reader to [26] for an analogous setup used to analyze the response matrix for extended targets in the free space.

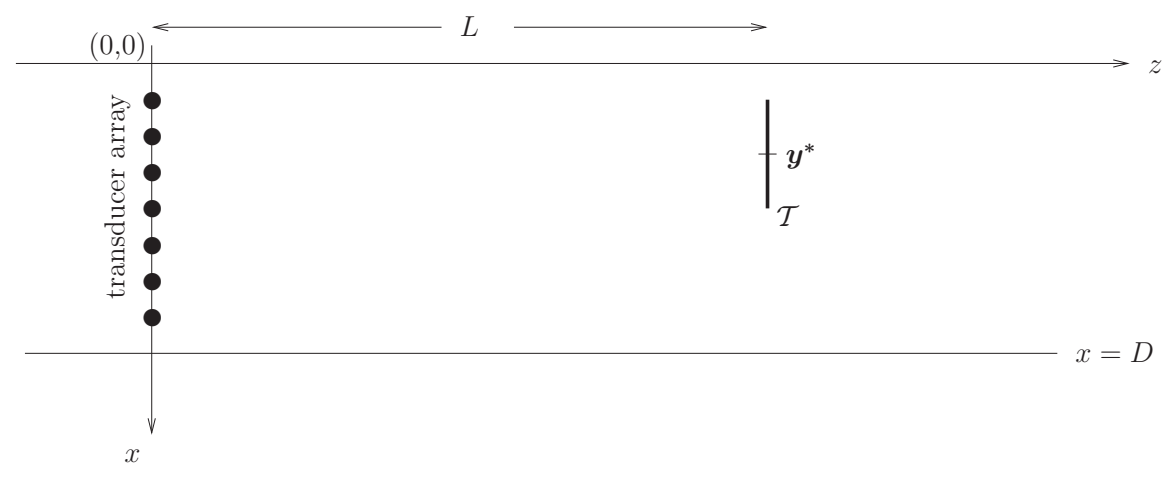

Figure 13. A vertical crack, implemented in the waveguide.

5.1. Array response matrix. Assuming unit reflectivity at each point of the target, we may approximate the response in a receiver placed at $\overrightarrow{\boldsymbol{x}}_{r}=\left(0, x_{r}\right)$ due to a source at $\overrightarrow{\boldsymbol{x}}_{s}=$ $\left(0, x_{s}\right), r, s \in\{1,2, \ldots, N\}$, as

$$
\widehat{\Pi}\left(\overrightarrow{\boldsymbol{x}}_{r}, \overrightarrow{\boldsymbol{x}}_{s}, \omega\right)=\int_{\mathcal{T}} \widehat{G}\left(\overrightarrow{\boldsymbol{y}}, \overrightarrow{\boldsymbol{x}}_{r}\right) \widehat{G}\left(\overrightarrow{\boldsymbol{y}}, \overrightarrow{\boldsymbol{x}}_{s}\right) d x
$$


where $\overrightarrow{\boldsymbol{y}}=(L, x), x \in\left[x_{0}-b / 2, x_{0}+b / 2\right]$, and $\widehat{G}$ is the Green's function defined in (2.5). For future reference, let us also recall that $\mu_{n}, X_{n}$ are the eigenvalues and corresponding eigenfunctions of the operator $-d^{2} / d x^{2}$ in $H^{2}(0, D) \cap H_{0}^{1}(0, D)$, defined in (2.3), and $\beta_{n}$ are the horizontal wavenumbers defined in (2.4).

Inserting (2.5) into (5.1) shows that

$$
\widehat{\Pi}\left(\overrightarrow{\boldsymbol{x}}_{r} ; \overrightarrow{\boldsymbol{x}}_{s}, \omega\right)=-\frac{1}{4} \sum_{m, n=1}^{\infty} \frac{\mathrm{e}^{\mathrm{i} \beta_{m} L}}{\beta_{m}} X_{m}\left(x_{s}\right) X_{n}\left(x_{r}\right) \frac{\mathrm{e}^{\mathrm{i} \beta_{n} L}}{\beta_{n}} \int_{x_{0}-\frac{b}{2}}^{x_{0}+\frac{b}{2}} X_{m}(x) X_{n}(x) d x .
$$

Moreover, let $g_{m}\left(x_{i}\right):=\frac{i}{2} \frac{e^{i \beta_{m} L}}{\beta_{m}} X_{m}\left(x_{i}\right)=\left(\widehat{G}\left(\cdot, \overrightarrow{\boldsymbol{x}}_{i}\right), X_{m}\right)_{L^{2}(\mathcal{C})}$, where the outer parentheses in the second equality denote the standard $L^{2}$ inner product on $\mathcal{C}$. Let us also define $\mathcal{G}$ to be the matrix

$$
\mathcal{G}:=\left(\begin{array}{cccccc}
g_{1}\left(x_{1}\right) & g_{2}\left(x_{1}\right) & \ldots & g_{M}\left(x_{1}\right) & g_{M+1}\left(x_{1}\right) & \ldots \\
g_{1}\left(x_{2}\right) & g_{2}\left(x_{2}\right) & \ldots & g_{M}\left(x_{2}\right) & g_{M+1}\left(x_{2}\right) & \ldots \\
\vdots & \vdots & & \vdots & \vdots & \\
g_{1}\left(x_{N}\right) & g_{2}\left(x_{N}\right) & \ldots & g_{M}\left(x_{N}\right) & g_{M+1}\left(x_{N}\right) & \ldots
\end{array}\right)
$$

and $A_{\text {inf }}$ the (infinite) matrix with entries

$$
a_{m n}=\int_{x_{0}-\frac{b}{2}}^{x_{0}+\frac{b}{2}} X_{m}(x) X_{n}(x) d x, \quad m, n=1,2, \ldots .
$$

Then, using (5.2), we may write the array response matrix $\widehat{\Pi}$ as a matrix product of the form

$$
\widehat{\Pi}=\mathcal{G} A_{\text {inf }} \mathcal{G}^{T} .
$$

We point out that if $L$ is sufficiently large and $m \geq M+1$, then

$$
g_{m}\left(x_{i}\right)=\frac{\mathrm{i}}{2} \frac{\mathrm{e}^{\mathrm{i} \beta_{m} L}}{\beta_{m}} X_{m}\left(x_{i}\right)=\frac{1}{2} \frac{\mathrm{e}^{-\sqrt{\mu_{m}-k^{2}} L}}{\sqrt{\mu_{m}-k^{2}}} X_{m}\left(x_{i}\right) \simeq 0,
$$

or, equivalently, only the principal $N \times M$ part of $\mathcal{G}$ is practically nonzero; thus, in practice, instead of $A_{\text {inf }}$ we work with its $M \times M$ principal part denoted by $A_{M}$. At this point let us remark that although $\widehat{\Pi}$ is associated to $A_{\text {inf }}$ (and, in practice, with $A_{M}$ ) through (5.4), we cannot infer from this formula an explicit relation between their singular values.

It is now natural to ask how $A_{M}$ is related to the matrix $\widehat{\mathbb{P}}$ that results when we project the array response matrix $\widehat{\Pi}$ onto the propagating modes. Recalling the definition of $\widehat{\mathbb{P}}(3.12)$ and using the orthonormality of the eigenfunctions $\left\{X_{n}\right\}_{n=1,2, \ldots}$, one may immediately see that

$$
\widehat{\mathbb{P}}_{m n}=-\frac{1}{4} \mathrm{e}^{\mathrm{i}\left(\beta_{m}+\beta_{n}\right) L} a_{m n}, \quad m, n=1, \ldots, M
$$

or, in matrix form,

$$
\widehat{\mathbb{P}}=-\frac{1}{4} Q A_{M} Q
$$

where $Q$ is the diagonal matrix $\operatorname{diag}\left(\mathrm{e}^{\mathrm{i} \beta_{1} L}, \ldots, \mathrm{e}^{\mathrm{i} \beta_{M} L}\right)$. Hence $\widehat{\mathbb{P}}$ is unitarily equivalent to $A_{M}$, since $Q^{*} Q=I$. 
5.2. Spectral properties of $\boldsymbol{A}_{M}$. In this section, we turn our attention to the spectral properties of the real symmetric matrix $A_{M}$, since we have shown that it is related to both matrices $\widehat{\Pi}$ and $\widehat{\mathbb{P}}$ involved in the computation of $\mathcal{I}^{\mathrm{KM}}$ and $\widetilde{\mathcal{I}}^{\mathrm{KM}}$, respectively. In what follows, for an arbitrary positive integer $n$ we shall denote by $A_{n}$ the principal $n \times n$ submatrix of $A_{\text {inf }}$, and we will refer to either the eigenvalues/eigenvectors of $A_{n}$ or to its singular values/vectors, since the latter are just the former written in descending order.

For $\ell, m \geq 1$, and in view of the simple trigonometric identity $2 \sin a \sin b=\cos (a-b)-$ $\cos (a+b)$, it holds that

$$
\begin{aligned}
a_{\ell m} & =\int_{x_{0}-\frac{b}{2}}^{x_{0}+\frac{b}{2}} X_{\ell}(x) X_{m}(x) d x=\frac{2}{D} \int_{x_{0}-\frac{b}{2}}^{x_{0}+\frac{b}{2}} \sin \frac{\ell \pi x}{D} \sin \frac{m \pi x}{D} d x \\
& =\frac{1}{D} \int_{x_{0}-\frac{b}{2}}^{x_{0}+\frac{b}{2}} \cos \frac{(\ell-m) \pi x}{D} d x-\frac{1}{D} \int_{x_{0}-\frac{b}{2}}^{x_{0}+\frac{b}{2}} \cos \frac{(\ell+m) \pi x}{D} d x .
\end{aligned}
$$

Hence

$$
A_{n}=T_{n}-H_{n}, \quad \text { where } T_{n}:=\left(t_{\ell-m}\right)_{\ell, m=1}^{n}, \quad H_{n}:=\left(t_{\ell+m}\right)_{\ell, m=1}^{n},
$$

and

$$
t_{m}=\frac{1}{D} \int_{0}^{D} \mathbb{1}_{\mathcal{T}}(x) \cos \frac{m \pi x}{D} d x
$$

where $\mathbb{1}_{\mathcal{T}}(x)$ is the indicator function of $\mathcal{T}$. Note that $\mathbb{1}_{\mathcal{T}}(x)$ is the so-called generating function of the matrices $A_{n}, T_{n}$, and $H_{n}$. One may immediately recognize $T_{n}$ as a (real symmetric) Toeplitz matrix, i.e., a matrix with constant entries along the diagonals, and $H_{n}$ as a Hankel matrix, i.e., a matrix with constant skew-diagonals (these are the diagonals that are perpendicular to the main diagonal). Hence $A_{n}$ is a Toeplitz-minus-Hankel matrix. As we shall briefly discuss next, the spectral properties of $A_{n}$ are determined by the Toeplitz part $T_{n}$. This can be seen, for example, by modifying appropriately the proofs in the work of Fasino [8], who studies the spectral properties of Toeplitz-plus-Hankel matrices, or by tracing back to the work of Trench [24], who studies the spectral properties of the real symmetric Toeplitz matrix

$$
T_{n}=\left(t_{r-s}\right)_{r, s=1}^{n}, \quad \text { where } t_{r}=\frac{1}{\pi} \int_{0}^{\pi} f(x) \cos r x d x,
$$

and the generating function $f \in L^{2}[0, \pi]$. Following Trench's notation, a vector $\boldsymbol{x} \in \mathbb{R}^{n}$ is called symmetric if $J \boldsymbol{x}=\boldsymbol{x}$ and skew-symmetric if $J \boldsymbol{x}=-\boldsymbol{x}$, where $J$ is the flip matrix (i.e., the matrix that has ones on the secondary diagonal and zeros elsewhere); see also [1]. Moreover, an eigenvalue $\lambda$ of $T$ is defined to be even (odd) if $T$ has a symmetric (skewsymmetric) $\lambda$-eigenvector.

Now, let $\lambda_{1}^{(n)} \leq \lambda_{2}^{(n)} \leq \cdots \leq \lambda_{n}^{(n)}$ be the eigenvalues of $T_{n}$, and let $\nu_{1}^{(n)} \leq \nu_{2}^{(n)} \leq \cdots \leq \nu_{n}^{(n)}$ be the eigenvalues of $A_{n}$. Since, in our case, the generating function $f$ of the matrices $A_{n}, T_{n}$, and $H_{n}$, is the indicator function of $\mathcal{T}$, its essential lower and upper bounds are simply 0 and 1, respectively. A result of Szegő (see [11, pp. 64-65]) guarantees that (i) $0 \leq \lambda_{i}^{(n)} \leq 1$ for all 
$i=1, \ldots, n$; (ii) for any fixed integer $k, \lambda_{k}^{(n)} \rightarrow 0, \lambda_{n-k}^{(n)} \rightarrow 1$ as $n \rightarrow \infty$; and (iii) if $G$ is any continuous function defined in $[0,1]$, we have

$$
\lim _{n \rightarrow \infty} \frac{1}{n} \sum_{i=1}^{n} G\left(\lambda_{i}^{(n)}\right)=\frac{1}{D} \int_{0}^{D} G(f(x)) d x .
$$

Moreover, the following theorem specializes results stated in [24] to our case, where we work on $[0, D]$, the entries of our matrix are given in (5.7), and $f=\mathbb{1}_{\mathcal{T}}$.

Theorem 5.1.

(a) The odd eigenvalues $\kappa_{1}^{(2 n+1)} \leq \kappa_{2}^{(2 n+1)} \leq \cdots \leq \kappa_{n}^{(2 n+1)}$ of $T_{2 n+1}$ are the eigenvalues of $A_{n}$ [24, Thm. 2].

(b) Since $f$ is bounded, the sets $\left\{\lambda_{i}^{(n)}\right\}_{i=1}^{n}$ and $\left\{\kappa_{i}^{(2 n+1)}\right\}_{i=1}^{n}$ are absolutely equally distributed [24, Def. 1 and Thm. 5].

(c) Since $f$ is bounded, (5.8) also holds for $\nu_{i}^{(n)}$ instead of $\lambda_{i}^{(n)}$. Moreover, if for $\epsilon>0$ $C(\epsilon, 1-\epsilon, n)$ is the cardinality of the set $\left\{i: \epsilon \leq \kappa_{i}^{(2 n+1)} \leq 1-\epsilon\right\}$, then

$$
\lim _{n \rightarrow \infty} \frac{1}{n} C(\epsilon, 1-\epsilon, n)=0
$$

[24, Lem. 2 and Thm. 6].

Summarizing, the eigenvalues of the matrix $A_{n}$ are clustered near 0 and 1 , and considering the function $G$ to be the identity on $[0,1]$, we immediately see that

$$
\lim _{n \rightarrow \infty} \frac{1}{n} \sum_{i=1}^{n} \nu_{i}^{(n)}=\frac{1}{D} \int_{0}^{D} \mathbb{1}_{\mathcal{T}}(x) d x=\frac{b}{D} .
$$

This indicates that asymptotically, as $n \rightarrow \infty$, the ratio of the nonzero eigenvalues of $A_{n}$ to the total number of eigenvalues is equal to $b / D$. In our case, where $n$ is equal to the number of propagating modes $M=\left\lfloor\frac{2 D}{\lambda}\right\rfloor$, it is expected that the number of "significant" singular values for our matrix $A_{M}$ is

$$
\left[M \frac{b}{D}\right] \approx\left[\frac{2 b}{\lambda}\right]
$$

To conclude, we have shown that the number of nonzero singular values is related to the size of the object. In particular, by performing a standard resolution analysis, one may deduce that the cross-range resolution is $\lambda / 2$; therefore the rank of the matrix is roughly equal to the size of the object divided by the "array resolution." The same result has been obtained in the free space case (see [4, sect. 4.5.2]).

In the next subsection we explore the form of the eigenvectors of $A_{M}$ in order to gain some insight about the behavior of the functionals that we are using for selective imaging.

5.3. Selective imaging. We consider the imaging functional $\widetilde{\mathcal{I}}_{J}^{\mathrm{KM}}$, where the subscript $J$ indicates that the matrix $\widehat{\mathbb{P}}$ is approximated by means of the $J$ th singular vector for selective imaging. Then, for a search point $\overrightarrow{\boldsymbol{y}}^{\mathrm{s}}=\left(L, x^{\mathrm{s}}\right)$ located at the correct range $L,(3.16)$ and (5.5) imply that

$$
\widetilde{\mathcal{I}}_{J}^{\mathrm{KM}}\left(\overrightarrow{\boldsymbol{y}}^{\mathrm{s}}\right)=\frac{1}{16 h^{2}} \sum_{m, n=1}^{M} X_{m}\left(x^{\mathrm{s}}\right) X_{n}\left(x^{\mathrm{s}}\right) \sigma_{J} u_{J}^{m} u_{J}^{n}=\sigma_{J}\left(\frac{1}{4 h} \sum_{n=1}^{M} u_{J}^{n} X_{n}\left(x^{\mathrm{s}}\right)\right)^{2},
$$



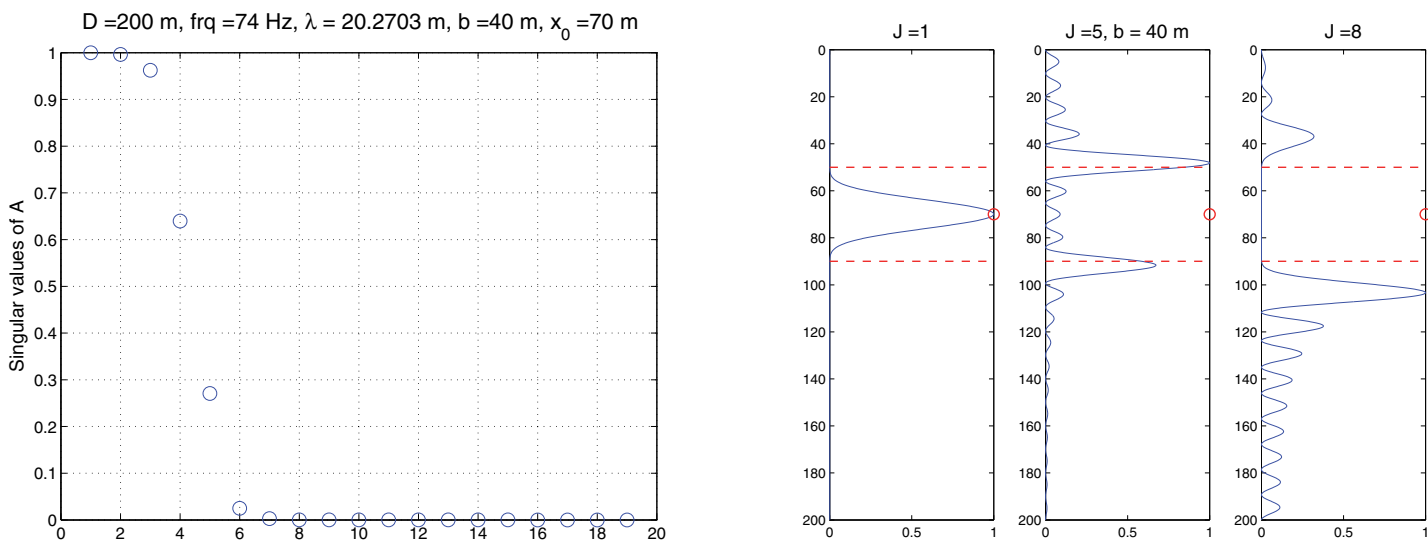

Figure 14. Left: The singular values of $A_{M}(M=19)$. Right: The graphs of $\left(s_{J}(x)\right)^{2}, x \in[0,200]$, for $J=1,5,8$, normalized with respect to their largest values.

where $\boldsymbol{u}_{J}=\left(u_{J}^{1}, u_{J}^{2}, \ldots, u_{J}^{M}\right)^{T}$ is the singular vector of $A_{M}$ that corresponds to the singular value $\sigma_{J}$. Recalling the definition of $X_{n}$ in (2.3), and suppressing constants, we associate to $\boldsymbol{u}_{J}$ the trigonometric polynomial

$$
s_{J}(x)=\sum_{n=1}^{M} u_{J}^{n} \sin \frac{n \pi x}{D} .
$$

Therefore, the behavior of $\widetilde{\mathcal{I}}_{J}^{\mathrm{KM}}$ is in fact determined by the properties of $s_{J}(x)$, which we shall illustrate in the following example: Consider a waveguide with depth equal to $D=200 \mathrm{~m}$ and constant sound speed equal to $c_{0}=1500 \mathrm{~m} / \mathrm{s}$. In Figure 14 (left subplot) we plot the singular values of $A_{M}$ for a frequency of $74 \mathrm{~Hz}$, for which the number of propagating modes $M=19$, the wavelength $\lambda \approx 20.27 \mathrm{~m}$, and $\mathcal{T}$ is centered at $x_{0}=70 \mathrm{~m}$, while its width is taken as $b=40 \mathrm{~m} \approx 2 \lambda$. According to (5.9) the number of "significant" singular values equals 4. Indeed, one may see that the first three remain very close to 1 , the fourth is approximately 0.65 , and the fifth lies in the transition layer between 0 and 1 close to 0.3 . The sixth is less than 0.1 , while the rest are very close to zero. In the right subplot of Figure 14 we plot the square of the values of the trigonometric polynomials $s_{J}(x)$ for $J=1,5$, and 8 , normalized with respect to their largest values. The relevant singular vectors $\boldsymbol{u}_{J}, J=1,5,8$, have been computed with MATLAB. The red circle in these graphs indicates the center $x_{0}$ of $\mathcal{T}$, and the red dashed lines the width $b$. Note that $s_{1}(x)$ that corresponds to the first singular value is supported in $\mathcal{T}$ and exhibits a peak at the center $x_{0}, s_{5}(x)$ exhibits peaks near the endpoints of $\mathcal{T}$, and $s_{8}(x)$ is approximately zero for $x \in \mathcal{T}$. These results remind us of the spectral properties and the band-limited behavior of the so-called prolate matrix; see [21, 25, 12]. As remarked in [12] for the prolate matrix, one may characterize the eigenspace corresponding to the eigenvalue cluster near 1 as the signal subspace, the eigenspace corresponding to the eigenvalue cluster near 0 as the noise subspace, and the eigenspace corresponding to eigenvalues in the intermediate layer as the transient subspace; the terminology is adopted from [12].

Next, we try to explore the form of the singular vectors (eigenvectors) of $A_{M}$. To this 
end, we consider an orthonormal basis $\left\{Y_{j}(x)\right\}_{j=1}^{\infty}$ of $L^{2}\left[x_{0}-b / 2, x_{0}+b / 2\right]$; specifically, let

$$
Y_{j}(x)=\sqrt{\frac{2}{b}} \sin \left(\frac{\left(x-x_{0}+\frac{b}{2}\right) j \pi}{b}\right), \quad j=1,2, \ldots
$$

Then, the restriction of the eigenfunctions $X_{n} \in L^{2}[0, D]$ on the crack $\mathcal{T}$ may be written as

$$
\left.X_{n}(x)\right|_{\left[x_{0}-b / 2, x_{0}+b / 2\right]}=\sum_{j=1}^{\infty} v_{j}^{n} Y_{j}(x), \quad \text { where } v_{j}^{n}=\int_{x_{0}-\frac{b}{2}}^{x_{0}+\frac{b}{2}} X_{n}(x) Y_{j}(x) d x .
$$

The orthonormality of the $Y_{i}$ and Parseval's relation imply that

$$
a_{m n}=\left(X_{m}, X_{n}\right)_{L^{2}(\mathcal{T})}=\sum_{i}\left(X_{m}, Y_{i}\right)_{L^{2}(\mathcal{T})}\left(X_{n}, Y_{i}\right)_{L^{2}(\mathcal{T})}=\sum_{i=1}^{\infty} v_{i}^{m} v_{i}^{n}
$$

Let us define

$$
\boldsymbol{v}_{i}=\left(v_{i}^{1}, v_{i}^{2}, \ldots, v_{i}^{M}, \ldots\right)^{T},
$$

and note that the sequences $\boldsymbol{v}_{i} \in l_{2}$ for every $i$. Then the infinite matrix $A_{\text {inf }}$ may be written as

$$
A_{\text {inf }}=\sum_{i=1}^{\infty} \boldsymbol{v}_{i} \boldsymbol{v}_{i}^{T}
$$

Moreover, the $\boldsymbol{v}_{i}$ are orthonormal. Indeed, let $\langle\cdot, \cdot\rangle$ denote the standard inner product in $l_{2}$, and $\widetilde{Y}_{i}$ the extension by zero of $Y_{i}$ on $[0, D]$. Then

$$
\begin{aligned}
&\left\langle\boldsymbol{v}_{i}, \boldsymbol{v}_{j}\right\rangle=\sum_{n=1}^{\infty} v_{i}^{n} v_{j}^{n}=\sum_{n}\left(X_{n}, Y_{i}\right)_{L^{2}(\mathcal{T})}\left(X_{n}, Y_{j}\right)_{L^{2}(\mathcal{T})} \\
&=\sum_{n}\left(\widetilde{Y}_{i}, X_{n}\right)_{L^{2}[0, D]}\left(\widetilde{Y}_{j}, X_{n}\right)_{L^{2}[0, D]} \\
& \stackrel{\text { Parseval }}{=}\left(\widetilde{Y}_{i}, \widetilde{Y}_{j}\right)_{L^{2}[0, D]}=\left(Y_{i}, Y_{j}\right)_{L^{2}(\mathcal{T})}=\delta_{i j} .
\end{aligned}
$$

Let $V$ be the closure of $\operatorname{span}\left\{\boldsymbol{v}_{i}\right\}_{i=1,2, \ldots}$. Then $l_{2}=V \oplus V^{\perp}$, and $A_{\text {inf }}$ is a projection operator whose eigenvalues are 0 and 1 .

It remains to investigate the relation between the eigenvectors $\boldsymbol{u}_{j}$ of $A_{M}$ that correspond to eigenvalues close to 1 , and the eigenvectors $\boldsymbol{v}_{j}$ of $A_{\text {inf }}$ corresponding to the eigenvalue 1 . Let us multiply, for example, $A_{M}$ by the vector consisting of the $M$ first components of $\boldsymbol{v}_{j}$ 

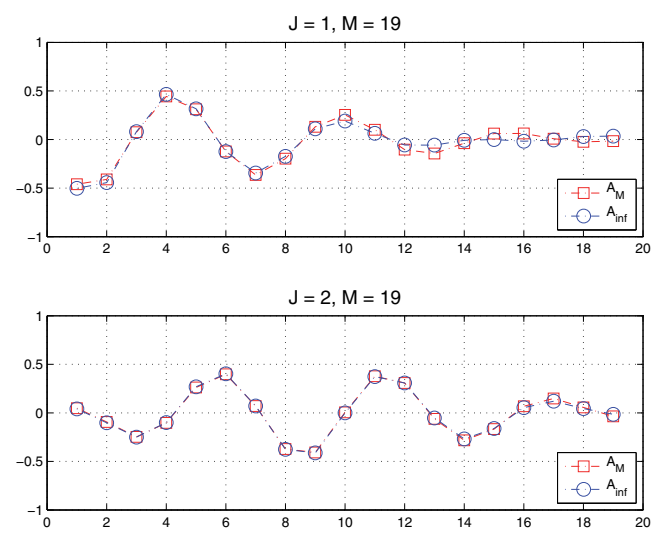

Figure 15. The singular vector $\boldsymbol{u}_{J}$ of $A_{M}$ (its components are marked with red squares) versus the $M$ first terms of $\boldsymbol{v}_{J}$ marked with blue circles for $J=1$ (top) and $J=2$ (bottom).

(i.e., $\left.\left(v_{j}^{1}, v_{j}^{2}, \ldots, v_{j}^{M}\right)^{T}\right)$. The $i$ th component of the resulting vector is equal to

$$
\begin{aligned}
\sum_{k=1}^{M} a_{i k} v_{j}^{k} & =\sum_{k=1}^{\infty} a_{i k} v_{j}^{k}-\sum_{k=M+1}^{\infty} a_{i k} v_{j}^{k} \\
& =\sum_{k=1}^{\infty}\left(X_{i}, X_{k}\right)_{L^{2}(\mathcal{T})}\left(X_{k}, Y_{j}\right)_{L^{2}(\mathcal{T})}-\sum_{k=M+1}^{\infty} a_{i k} v_{j}^{k} \\
& =\sum_{k=1}^{\infty}\left(X_{i} \mathbb{1}_{\mathcal{T}}, X_{k}\right)_{L^{2}[0, D]}\left(\tilde{Y}_{j}, X_{k}\right)_{L^{2}[0, D]}-\sum_{k=M+1}^{\infty} a_{i k} v_{j}^{k} \\
& =\left(X_{i} \mathbb{1}_{\mathcal{T}}, \tilde{Y}_{j}\right)_{L^{2}[0, D]}-\sum_{k=M+1}^{\infty} a_{i k} v_{j}^{k}=v_{j}^{i}-\left(\sum_{k=M+1}^{\infty} a_{i k} v_{j}^{k}\right) .
\end{aligned}
$$

The last term in parentheses in the above equation depends on $i, j, b$, and $D$, and is in general small (this has been checked numerically in all the test cases that we have considered). Hence if the $j$ th singular value is approximately 1 , then $\left(v_{j}^{1}, v_{j}^{2}, \ldots, v_{j}^{M}\right)^{T}$ approximates $\boldsymbol{u}_{j}$, i.e., the $j$ th singular vector of $A_{M}$. This is illustrated in Figure 15, where we plot the components of the first two singular vectors $\boldsymbol{u}_{J}, J=1,2$, of $A_{M}$ and the $M$ first terms of the sequences $\boldsymbol{v}_{J}$, $J=1,2$, for the parameters of the previous example for which $M=19$.

Hence, as long as $J$ is associated to a singular vector that lies in the signal subspace, one may approximate $\widetilde{\mathcal{I}}_{J}^{\mathrm{KM}}$ as

$$
\widetilde{\mathcal{I}}_{J}^{\mathrm{KM}}\left(\overrightarrow{\boldsymbol{y}}^{\mathrm{s}}\right) \approx \sum_{m, n=1}^{M} X_{m}\left(x^{\mathrm{s}}\right) X_{n}\left(x^{\mathrm{s}}\right) v_{J}^{m} v_{J}^{n}=\left(\sum_{n=1}^{M} v_{J}^{n} X_{n}\left(x^{\mathrm{s}}\right)\right)^{2},
$$


where constants are once again suppressed. Moreover, for $M$ large enough, we formally get

$$
\begin{aligned}
\left(\widetilde{\mathcal{I}}_{J}^{\mathrm{KM}}\left(\overrightarrow{\boldsymbol{y}}^{\mathrm{s}}\right)\right)^{1 / 2} & \approx\left|\sum_{n=1}^{\infty} v_{J}^{n} X_{n}\left(x^{\mathrm{s}}\right)\right|=\left|\sum_{n}\left(X_{n}, Y_{J}\right)_{L^{2}(\mathcal{T})} X_{n}\left(x^{\mathrm{s}}\right)\right| \\
& =\left|\sum_{n}\left(\widetilde{Y}_{J}, X_{n}\right)_{L^{2}[0, D]} X_{n}\left(x^{\mathrm{s}}\right)\right|=\left|\widetilde{Y}_{J}\left(x^{\mathrm{s}}\right)\right| .
\end{aligned}
$$

Using the specific form of the $Y_{J}$ we deduce that, as long as the $J$ th singular value is close to 1 ,

$$
\widetilde{\mathcal{I}}_{J}^{\mathrm{KM}}\left(\overrightarrow{\boldsymbol{y}}^{\mathrm{s}}\right) \approx \frac{2}{b} \sin ^{2}\left(\frac{\left(x^{\mathrm{s}}-x_{0}+\frac{b}{2}\right) J \pi}{b}\right) .
$$

In the next section, we will compare these asymptotic results with numerical simulations.

5.4. Numerical experiments. In this section, we present numerical results for the simplified model of the vertical one-dimensional scatterer (the crack). Specifically, (a) we compare results of selective imaging with $\widetilde{\mathcal{I}}_{J}^{\mathrm{KM}}$ with those obtained using the asymptotic formulas (5.15), (5.17), and (b) we compare results between selective imaging with $\mathcal{I}_{J}^{\mathrm{KM}}$ and $\widetilde{\mathcal{I}}_{J}^{\mathrm{KM}}$. In all the following examples the sound speed is taken as $c_{0}=1500 \mathrm{~m} / \mathrm{s}$, and the depth of the waveguide is equal to $D=200 \mathrm{~m}$.

5.4.1. $\tilde{\mathcal{I}}_{J}^{\mathrm{KM}}$ versus asymptotics. First, we consider a single frequency $f=74 \mathrm{~Hz}$; hence the wavelength $\lambda \approx 20.27 \mathrm{~m}$. The array has $N=39$ receivers, the pitch $h=5 \mathrm{~m} \simeq \lambda / 4$, and the crack, centered at $\left(L, x_{0}\right)=(410,70) \mathrm{m}$, has length $b=40 \mathrm{~m} \simeq 2 \lambda$. The number of propagating modes in the waveguide is $M=19$. The singular values of $A_{M}$ are shown in Figure 14. We have seen previously that $A_{M}$ and, consequently, the matrix $\widehat{\mathbb{P}}$ defined in (5.5) have $[2 b / \lambda]=4$ significant singular values. In other words, and with reference to Figure 14 , it is expected that the first three singular vectors compose the signal subspace, the fourth and fifth lie in the transient subspace, and the rest correspond to the noise subspace.

We now turn to checking the validity of the asymptotic expressions (5.15) and (5.17). In Figure 16 we superimpose the normalized graphs of $\widetilde{\mathcal{I}}_{J}^{\mathrm{KM}}\left(\overrightarrow{\boldsymbol{x}}^{\mathrm{s}}, \omega\right)$ (see (3.16)), of (5.15), and of (5.17) for $\overrightarrow{\boldsymbol{x}}^{\mathrm{s}}$ that are located at the correct range $L$, and for $J=1$ and 2 . The subscript $J$ indicates that only the $J$ th singular vector is employed in the filtered version of the matrix $\widehat{\mathbb{P}}$, defined in (5.5). As one may immediately see, there is good agreement between the numerics and the asymptotic expressions.

In Figure 17 we show analogous results for selective imaging with $\widetilde{\mathcal{I}}_{J}^{\mathrm{KM}}\left(\overrightarrow{\boldsymbol{x}}^{\mathrm{s}}, \omega\right)$ and (5.15), based on the third to the sixth singular vectors. Now, one may notice somewhat larger discrepancies, but, in general, the asymptotic expression agrees well with the numerical results for $J=3,4$, and 5 . For $J=6$ there is a clear mismatch, with $\widetilde{\mathcal{I}}_{J}^{\mathrm{KM}}$ focusing approximately $4.5 \mathrm{~m}$ below the lower endpoint of the crack, since now the corresponding singular vector belongs to the noise subspace. (The discrepancies, as expected, are even larger between the results of $\widetilde{\mathcal{I}}_{J}^{\mathrm{KM}}\left(\overrightarrow{\boldsymbol{x}}^{\mathrm{s}}, \omega\right)$ and those of $(5.17)$, and we do not include them in the figure. These discrepancies may be attributed to the fact that the asymptotic analysis is based on matrices of order $n$, where $n \rightarrow \infty$, while in the numerics only their principal $M \times M$ part has been 

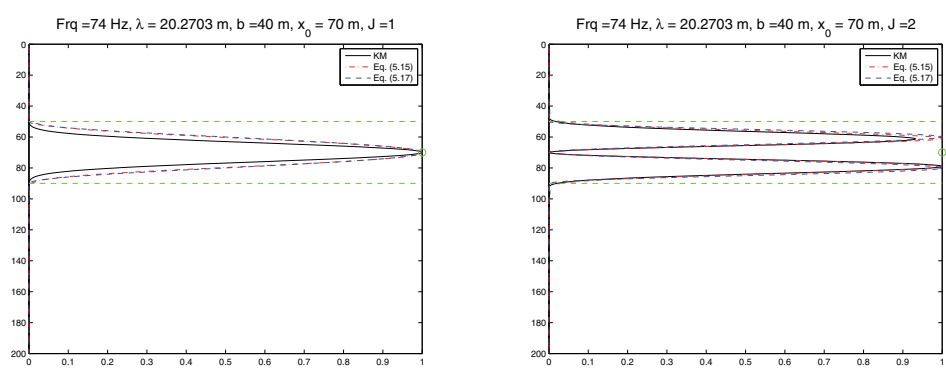

Figure 16. The graph of $\widetilde{\mathcal{I}}_{J}^{\mathrm{KM}}\left(\left(L, x^{\mathrm{s}}\right), \omega\right)$ (solid black line) normalized and superimposed on the graphs of (5.15) (dash-dotted red line) and (5.17) (dashed blue line). J indicates projection onto the J th singular vector. The ordinates $x_{0}-b / 2$ and $x_{0}+b / 2$ are indicated by dashed green lines, and $x_{0}$ is marked with a green circle. Here $c_{0}=1500 \mathrm{~m} / \mathrm{s}, f=74 \mathrm{~Hz}, D=200 \mathrm{~m}, b=40 \mathrm{~m}, x_{0}=70 \mathrm{~m}$, and $J=1$ (left subplot), $J=2$ (right subplot).
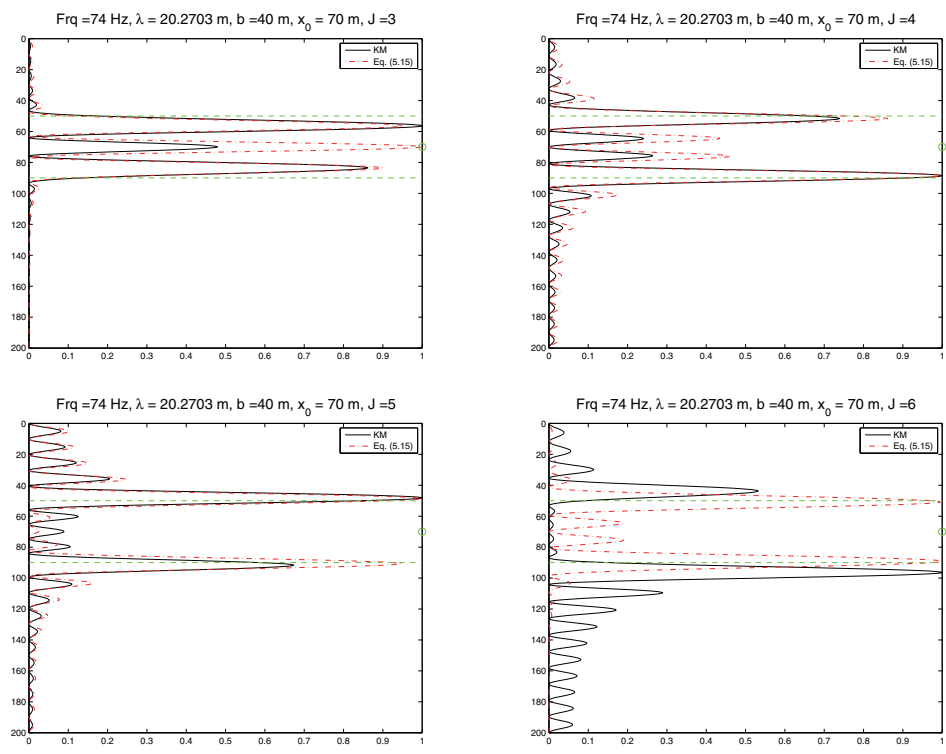

Figure 17. The graph of $\widetilde{\mathcal{I}}_{J}^{\mathrm{KM}}\left(\left(L, x^{\mathrm{s}}\right), \omega\right)$ (solid black line) superimposed on the graph of (5.15) (dashdotted red line). $J$ indicates projection onto the $J$ th singular vector, and the ordinates $x_{0}-b / 2$ and $x_{0}+b / 2$ are indicated by dashed green lines, while $x_{0}$ is marked with a green circle. Here $J=3,4,5$, and $6, c_{0}=1500 \mathrm{~m} / \mathrm{s}$, $f=74 \mathrm{~Hz}, \mathrm{D}=200 \mathrm{~m}, \mathrm{~b}=40 \mathrm{~m}$, and $x_{0}=70 \mathrm{~m}$.

taken into account. For example, the orthogonality of the $\boldsymbol{v}_{i}$ (see (5.13)) is valid only for the infinite matrix $A_{\text {inf }}$ and is expected to hold only approximately for fixed $n$.)

Figures 16 and 17 suggest that selective imaging with $\widetilde{\mathcal{I}}_{J}^{\mathrm{KM}}(\omega)$ by means of the first singular vector focuses at the middle of the object, while the fourth and fifth singular vectors are focusing mainly at the lower and the upper endpoints of the crack, respectively.

5.4.2. $\mathcal{I}_{J}^{\mathrm{KM}}$ versus $\tilde{\mathcal{I}}_{J}^{\mathrm{KM}}$. Here, we compare selective imaging with $\mathcal{I}_{J}^{\mathrm{KM}}$ versus $\widetilde{\mathcal{I}}_{J}^{\mathrm{KM}}$, keeping the same setup as in section 5.4.1. 


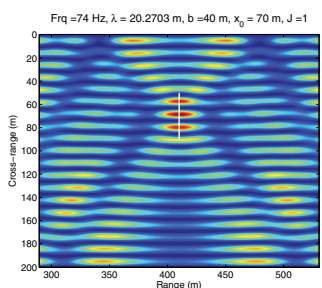

Frea $=74 \mathrm{~Hz}, \lambda=20.2703 \mathrm{~m}, \mathrm{~b}=40 \mathrm{~m}, \mathrm{x}_{\mathrm{u}}=70 \mathrm{~m}, \mathrm{~J}=1$

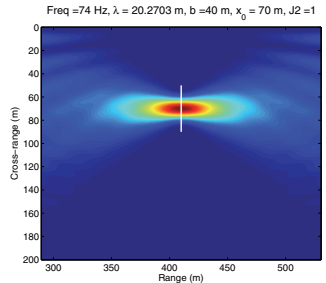

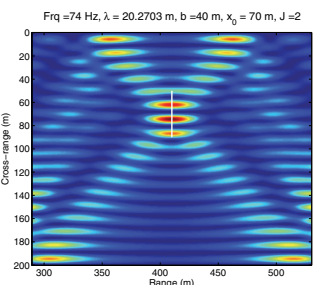

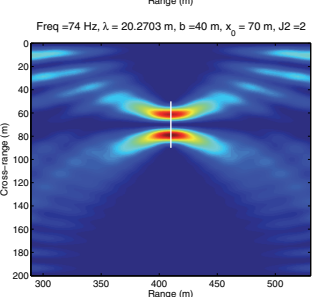

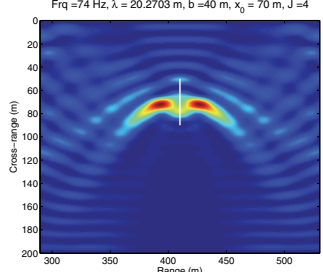

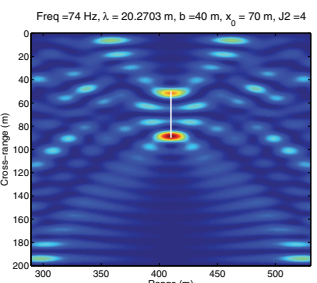

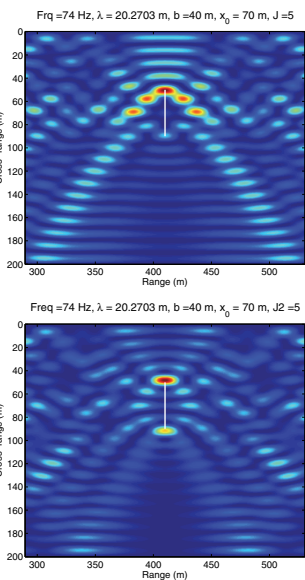

Figure 18. Values of $\mathcal{I}_{J}^{\mathrm{KM}}(\omega)$ (top row) and $\widetilde{\mathcal{I}}_{J}^{\mathrm{KM}}(\omega)$ (bottom row) for the crack, where $J=1,2,4,5$ indicates projection onto the $J$ th singular vector.

In the top row of Figure 18 we plot the modulus of $\mathcal{I}_{J}^{\mathrm{KM}}(\omega)$ (see (3.6)) for $J=1,2,4$, and 5 , and in the bottom row the corresponding results for the modulus of $\widetilde{\mathcal{I}}_{J}^{\mathrm{KM}}(\omega)$ (see (3.16)). As before, $J$ indicates projection onto the $J$ th singular vector, and the search domain is $[290,530] \times[0,200]$ (all distances are in meters). Note the robustness in the results of $\widetilde{\mathcal{I}}_{J}^{\mathrm{KM}}(\omega)$, where projection onto the first singular vector focuses in the center of the crack as projection onto subsequent singular vectors results in focusing towards the edges, as opposed to the results of $\mathcal{I}_{J}^{\mathrm{KM}}(\omega)$.

In this section we presented the theoretical analysis of our selective imaging functional, as well as numerical results for the model problem of a crack. These results are consistent with, and have helped in understanding and explaining, the numerical results presented in section 4 in the more general case of extended reflectors.

6. Conclusions. In this paper we considered the problem of selective imaging extended reflectors in a waveguide using an active array of sensors. To this end, we proposed a novel selective imaging functional based on Kirchhoff migration and the singular value decomposition of $\widehat{\mathbb{P}}(\omega)$, which is a weighted modal projection of the array response matrix. The proposed imaging method has been theoretically analyzed for a simplified model of a vertical one-dimensional reflector of width $b$, and the following main results were derived:

1. We showed that the rank of $\widehat{\mathbb{P}}(\omega)$ is equal to $\left[\frac{b}{\lambda / 2}\right]$, that is, the size of the reflector divided by the array resolution $\lambda / 2$ ( $\lambda$ being the wavelength at frequency $\omega)$. This is a novel result for a waveguide geometry.

2. We derived analytic expressions for the singular vectors of $\widehat{\mathbb{P}}(\omega)$ and for our selective imaging functional. Exploiting these expressions, we showed that imaging using the projection of $\widehat{\mathbb{P}}(\omega)$ onto its first singular vector exhibits focusing at the center of the reflector, while by projecting onto the last significant singular vector we create an image that focuses at the endpoints of the reflector.

These results are in agreement with those obtained in free space (cf. [4]). Moreover, they are consistent with, and explain our selective imaging numerical results concerning, several 
extended reflector geometries.

Acknowledgments. We would like to thank Dr. Adrien Semin for his invaluable help with Montjoie and Prof. M. Papadimitrakis for fruitful discussions on Toeplitz operators.

\section{REFERENCES}

[1] A. L. Andrew, Eigenvectors of certain matrices, Linear Algebra Appl., 7 (1973), pp. 151-162.

[2] J.-P. Berenger, A perfectly matched layer for the absorption of electromagnetic waves, J. Comput. Phys., 114 (1994), pp. 185-200.

[3] N. Bleistein, J. K. Cohen, and J. W. Stockwell, Jr., Mathematics of Multidimensional Seismic Imaging, Migration, and Inversion, Interdiscip. Appl. Math. 13, Geophysics and Planetary Sciences, Springer-Verlag, New York, 2001.

[4] L. Borcea, G. Papanicolaou, and F. Guevara Vasquez, Edge illumination and imaging of extended reflectors, SIAM J. Imaging Sci., 1 (2008), pp. 75-114.

[5] L. Borcea, G. Papanicolaou, and C. Tsogka, Optimal waveform design for array imaging, Inverse Problems, 23 (2007), pp. 1973-2020.

[6] L. Borcea, G. PAPAnicolaou, and C. Tsogka, Optimal illumination and waveform design for imaging in random media, J. Acoust. Soc. Am., 122 (2007), pp. 3507-3518.

[7] L. Borcea, G. Papanicolaou, and C. Tsogka, Subspace projection filters for imaging in random media, Comptes Rendus Mécanique, 338 (2010), pp. 390-401.

[8] D. Fasino, Spectral properties of Toeplitz-plus-Hankel matrices, Calcolo, 33 (1996), pp. 87-98.

[9] S. D. GEDNEy, An anisotropic perfectly matched layer-absorbing medium for the truncation of FDTD lattices, IEEE Trans. Antennas and Propagation, 44 (1996), pp. 1630-1639.

[10] G. H. Golub and C. F. Van Loan, Matrix Computations, The Johns Hopkins University Press, Baltimore, MD, 1996.

[11] U. Grenander And G. Szegő, Toeplitz Forms and Their Applications, Chelsea Publishing, New York, 1984.

[12] M. Hanke And J. Nagy, Inverse Toeplitz preconditioners for ill-posed problems, Linear Algebra Appl., 284 (1998), pp. $137-156$.

[13] C. Hazard And K. Ramdani, Selective acoustic focusing using time-harmonic reversal mirrors, SIAM J. Appl. Math., 64 (2004), pp. 1057-1076.

[14] S. Hou, K. Solna, And H. ZhaO, A direct imaging algorithm for extended targets, Inverse Problems, 22 (2006), pp. 1151-1178.

[15] F. B. Jensen, W. A. Kuperman, M. B. Porter, and H. Schmidt, Computational Ocean Acoustics, Springer-Verlag, New York, 2004.

[16] W. A. Kuperman And D. Jackson, Ocean acoustics, matched-field processing and phase conjugation, in Imaging of Complex Media with Acoustic and Seismic Waves, Topics Appl. Phys. 84, M. Fink, W. A. Kuperman, J.-P. Montagner, and A. Tourin, eds., Springer-Verlag, Berlin, Heidelberg, 2002, pp. 43-97.

[17] N. Mordant, C. Prada, And M. Fink, Highly resolved detection in a waveguide with the D.O.R.T. method, J. Acoust. Soc. Am., 105 (1999), pp. 2634-2642.

[18] B. Pinçon And K. Ramdani, Selective focusing on small scatterers in acoustic waveguides using time reversal mirrors, Inverse Problems, 23 (2007), pp. 1-25.

[19] C. Prada, J. De Rosny, D. Clorennec, J. G. Minonzio, A. Aubry, M. Fink, L. Bernière, S. Hibral, P. Billand, AND T. FolÉGot, Experimental detection and focusing in shallow water by decomposition of the time reversal operator, J. Acoust. Soc. Am., 122 (2007), pp. 761-768.

[20] C. Prada And M. Fink, Eigenmodes of the time reversal operator: A solution to selective focusing in multiple-target media, Wave Motion, 20 (1994), pp. 151-163.

[21] D. Slepian, Prolate spheroidal wave functions, Fourier analysis and uncertainty. V: The discrete case, Bell System Tech. J., 57 (1978), pp. 1371-1430.

[22] M. Tanter, J. F. Aubry, J. Gerber, J. L. Thomas, and M. Fink, Optimal focusing by spatiotemporal inverse filter. I. Basic principles, J. Acoust. Soc. Am., 110 (2001), pp. 37-47. 
[23] M. Tanter, J. L. Thomas, and M. Fink, Time reversal and the inverse filter, J. Acoust. Soc. Am., 108 (2000), pp. 223-234.

[24] W. F. Trench, Asymptotic distribution of the even and odd spectra of real symmetric Toeplitz matrices, Linear Algebra Appl., 302/303 (1999), pp. 155-162.

[25] J. M. VARAH, The prolate matrix, Linear Algebra Appl., 187 (1993), pp. 269-278.

[26] H. Zhaо, Analysis of the response matrix for an extended target, SIAM J. Appl. Math., 64 (2004), pp. 725-745. 